\title{
Principal Component Analysis in ECG Signal Processing
}

\author{
Francisco Castells, ${ }^{1}$ Pablo Laguna, ${ }^{2}$ Leif Sörnmo, ${ }^{3}$ Andreas Bollmann, ${ }^{4}$ and José Millet Roig ${ }^{5}$ \\ ${ }^{1}$ Grupo de Investigación en Bioingenería, Electrónica y Telemedicina, Departamento de Ingenería Electrónica, \\ Escuela Politécnica Superior de Gandía, Universidad Politécnica de Valencia (UPV), Ctra. Nazaret-Oliva, \\ 46730 Gandía, Spain \\ ${ }^{2}$ Communications Technology Group, Aragón Institute of Engineering Research, University of Zaragoza, \\ 50018 Zaragoza, Spain \\ ${ }^{3}$ Signal Processing Group, Department of Electrical Engineering, Lund University, 22100 Lund, Sweden \\ ${ }^{4}$ Department of Cardiology, Otto-von-Guericke-University Magdeburg, 39120 Magdeburg, Germany \\ ${ }^{5}$ Grupo de Investigación en Bioingenería, Electrónica y Telemedicina, Departamento de Ingenería Electrónica, \\ Universidad Politécnica de Valencia, Cami de Vera, 46022 Valencia, Spain
}

Received 11 May 2006; Revised 20 November 2006; Accepted 20 November 2006

Recommended by William Allan Sandham

\begin{abstract}
This paper reviews the current status of principal component analysis in the area of ECG signal processing. The fundamentals of PCA are briefly described and the relationship between PCA and Karhunen-Loève transform is explained. Aspects on PCA related to data with temporal and spatial correlations are considered as adaptive estimation of principal components is. Several ECG applications are reviewed where PCA techniques have been successfully employed, including data compression, ST-T segment analysis for the detection of myocardial ischemia and abnormalities in ventricular repolarization, extraction of atrial fibrillatory waves for detailed characterization of atrial fibrillation, and analysis of body surface potential maps.
\end{abstract}

Copyright (c) 2007 Francisco Castells et al. This is an open access article distributed under the Creative Commons Attribution License, which permits unrestricted use, distribution, and reproduction in any medium, provided the original work is properly cited.

\section{INTRODUCTION}

Principal component analysis (PCA) is a statistical technique whose purpose is to condense the information of a large set of correlated variables into a few variables ("principal components"), while not throwing overboard the variability present in the data set [1]. The principal components are derived as a linear combination of the variables of the data set, with weights chosen so that the principal components become mutually uncorrelated. Each component contains new information about the data set, and is ordered so that the first few components account for most of the variability. In signal processing applications, PCA is performed on a set of time samples rather than on a data set of variables. When the signal is recurrent in nature, like the ECG signal, the analysis is often based on samples extracted from the same segment location of different periods of the signal.

Signal processing is today found in virtually any system for ECG analysis, and has clearly demonstrated its importance for achieving improved diagnosis of a wide variety of cardiac pathologies. Signal processing is employed to deal with diverse issues in ECG analysis such as data compression, beat detection and classification, noise reduction, signal separation, and feature extraction. Principal component analysis has become an important tool for successfully addressing many of these issues, and was first considered for the purpose of efficient storage retrieval of ECGs. Over the years, this issue has remained central as a research topic, although the driving force has gradually changed from having been tiny hard disks to become slow transmission links. Noise reduction may be closely related to data compression as reconstruction of the original signal usually involves a set of eigenvectors whose noise level is low, and thus the reconstructed signal becomes low noise; such reduction is, however, mostly effective for noise with muscular origin. Classification of waveform morphologies in arrhythmia monitoring is another early application of PCA, in which a subset of the principal components serves as features which are used to distinguish between normal sinus beats and abnormal waveforms such as premature ventricular beats.

A recent application of PCA in ECG signal processing is robust feature extraction of various waveform properties for 
the purpose of tracking temporal changes due to myocardial ischemia. Historically, such tracking has been based on local measurements derived from the ST-T segment, however, such measurements are unreliable when the analyzed signal is noisy. With correlation as the fundamental signal processing operation, it has become clear that the use of principal components offer a more robust and global approach to the characterization of the ST-T segment. Signal separation during atrial fibrillation is another recent application of PCA, the specific challenge being to extract the atrial activity so that the characteristics of this common arrhythmia can be studied without interference from ventricular activity. Such separation is based on the fact that the two activities originate from different bioelectrical sources; separation may exploit temporal redundancy among successive heartbeats as well as spatial redundancy when multilead recordings are analyzed.

The purpose of the present paper is to provide an overview of PCA in ECG signal processing. Section 2 contains a brief description of PCA fundamentals and an explanation of the relationship between PCA and Karhunen-Loève transform (KLT). The remaining sections of the paper are devoted to the use of PCA in ECG applications, and touch upon possibilities and limitations when applying this technique. The present overview is confined to those particular applications where the output of PCA, or the KLT, is considered, whereas applications involving general eigenanalysis of a data matrix are left out. The latter type of applications include singular-value-decomposition-(SVD)-based techniques for ECG noise reduction and extraction of the fetal ECG [2-7]. Another such application is the measurement of repolarization heterogeneity in terms of $\mathrm{T}$ wave loop morphology, where the ratio between the two most significant eigenvalues has been incorrectly denoted as PCA ratio, see, for example, $[8,9]$.

\section{METHODS}

Principal component analysis in ECG signal processing takes its starting point from the samples of a segment located in some suitable part of the heartbeat. The location within the beat differs from one application to another and may involve the entire heartbeat or a particular activity such as the $\mathrm{P}$ wave, the QRS complex, or the T wave. Before the samples of a segment can be extracted, however, a fiducial point must be determined so that the exact segment location within the beat can be defined. Information on the fiducial point is typically provided by a QRS detector and, sometimes, in combination with a subsequent algorithm for wave delineation [10]. Accurate time alignment of the different segments is a key point in PCA, and special care must be taken when performing this step.

The signal segment of a beat is represented by the column vector

$$
\mathbf{x}=\left[\begin{array}{c}
x(1) \\
x(2) \\
\vdots \\
x(N)
\end{array}\right]
$$

where $N$ is the number of samples of the segment. The segment is often extracted from several successive beats, thus resulting in an ensemble of $M$ beats. The entire ensemble is compactly represented by the $N \times M$ data matrix,

$$
\mathbf{X}=\left[\begin{array}{llll}
\mathbf{x}_{1} & \mathbf{x}_{2} & \cdots & \mathbf{x}_{M}
\end{array}\right] .
$$

The beats $\mathbf{x}_{1}, \ldots, \mathbf{x}_{M}$ can be viewed as $M$ observations of the random process $\mathbf{x}$. While this formulation suggests that all beats considered originate from one patient, the beats may alternatively originate from a set of patients depending on the purpose of the analysis.

\subsection{Principal component analysis}

The derivation of principal components is based on the assumption that the signal $\mathbf{x}$ is a zero-mean random process being characterized by the correlation $\mathbf{R}_{x}=E\left[\mathbf{x x}^{T}\right]$. The principal components of $\mathbf{x}$ result from applying an orthonormal linear transformation $\Psi=\left[\begin{array}{llll}\psi_{1} & \psi_{2} & \cdots & \psi_{N}\end{array}\right]$ to $\mathbf{x}$,

$$
\mathbf{w}=\Psi^{T} \mathbf{x}
$$

so that the elements of the principal component vector $\mathbf{w}=$ $\left[\begin{array}{llll}w_{1} & w_{2} & \cdots & w_{N}\end{array}\right]^{T}$ become mutually uncorrelated. The first principal component is obtained as a scalar product $w_{1}=$ $\psi_{1}^{T} \mathbf{x}$, where the vector $\psi_{1}$ is chosen so that the variance of $w_{1}$,

$$
E\left[w_{1}^{2}\right]=E\left[\boldsymbol{\psi}_{1}^{T} \mathbf{x} \mathbf{x}^{T} \psi_{1}\right]=\psi_{1}^{T} \mathbf{R}_{x} \psi_{1}
$$

is maximized subject to the constraint that $\psi_{1}^{T} \psi_{1}=1$. The maximal variance is obtained when $\psi_{1}$ is chosen as the normalized eigenvector corresponding to the largest eigenvalue of $\mathbf{R}_{x}$, as denoted $\lambda_{1}$; the resulting variance is

$$
E\left[w_{1}^{2}\right]=\boldsymbol{\psi}_{1}^{T} \mathbf{R}_{x} \boldsymbol{\psi}_{1}=\lambda_{1} \boldsymbol{\psi}_{1}^{T} \boldsymbol{\psi}_{1}=\lambda_{1}
$$

Subject to the constraint that $w_{1}$ and the second principal component $w_{2}$ should be uncorrelated, $w_{2}$ is obtained by choosing $\psi_{2}$ as the eigenvector corresponding to the second largest eigenvalue of $\mathbf{R}_{x}$, and so on until the variance of $\mathbf{x}$ is completely represented by $\mathbf{w}$. Accordingly, to obtain the whole set of $N$ different principal components, the eigenvector equation for $\mathbf{R}_{x}$ needs to be solved,

$$
\mathbf{R}_{x} \Psi=\Psi \Lambda
$$

where $\boldsymbol{\Lambda}$ denotes a diagonal matrix with the eigenvalues $\lambda_{1}, \ldots, \lambda_{N}$. Since $\mathbf{R}_{x}$ is rarely known in practice, the $N \times N$ sample correlation matrix, defined by

$$
\hat{\mathbf{R}}_{x}=\frac{1}{M} \mathbf{X X}^{T},
$$

replaces $\mathbf{R}_{x}$ when the eigenvectors are calculated in (6).

Applying PCA to an ensemble of beats $\mathbf{X}$, the associated pattern of principal components reflects the degree of morphologic beat-to-beat variability: when the eigenvalue associated to the first principal component is much larger 
than those associated to other components, the ensemble exhibits a low morphologic variability, whereas a slow fall-off of the principal component values indicates a large variability. In most applications, the main goal of PCA is to concentrate the information of $\mathbf{x}$ into a subset of components, that is, $w_{1}, \ldots, w_{K}$, where $K<N$, while retaining the physiological information (note that typically $M \gg N$, otherwise $K<\min (N, M))$. The choice of $K$ may be guided by various statistical performance indices [1], of which one index is the degree of variation $\mathcal{R}_{K}$, reflecting how well the subset of $K$ principal components approximates the ensemble in energy terms,

$$
\mathcal{R}_{K}=\frac{\sum_{k=1}^{K} \lambda_{k}}{\sum_{k=1}^{N} \lambda_{k}} .
$$

In practice, however, $K$ is usually chosen so that the performance is clinically acceptable and that no vital signal information is lost.

The above derivation results in principal components that characterize intrabeat correlation. However, it is equally useful to define an $M \times M$ sample correlation matrix

$$
\hat{\mathbf{R}}_{x}^{\bullet}=\frac{1}{N} \mathbf{X}^{T} \mathbf{X},
$$

in order to characterize interbeat correlation. In this case, the principal components are computed for each sample $n$ rather than for every beat as was done in (3),

$$
\mathbf{w}(n)=\Psi^{\bullet T} \mathbf{x}(n)
$$

where

$$
\mathbf{x}(n)=\left[\begin{array}{c}
x_{1}(n) \\
x_{2}(n) \\
\vdots \\
x_{M}(n)
\end{array}\right]
$$

and $\Psi^{\bullet T}$ is the eigenvector matrix of $\hat{\mathbf{R}}_{x}^{\bullet}$.

Figure 1 illustrates the properties of the two types of sample correlation matrices in (7) and (9), respectively, by presenting the related eigenvalues and eigenvectors and the resulting principal components. The analyzed signal is a singlelead ECG which has been converted into a data matrix $\mathbf{X}$ so that each of its columns contains one beat, beginning just before the $\mathrm{P}$ wave.

When $M \ll N$, it is much faster to diagonalize $\hat{\mathbf{R}}_{x}^{\bullet}$ than $\hat{\mathbf{R}}_{x}$. This property can be realized by premultiplying both sides of

$$
\mathbf{X}^{T} \mathbf{X} \psi_{k}^{\bullet}=\lambda_{k} \psi_{k}^{\bullet}
$$

by $\mathbf{X}$, thus yielding

$$
\mathbf{X X}^{T} \mathbf{X} \psi_{k}^{\bullet}=\lambda_{k} \mathbf{X} \psi_{k}^{\bullet}
$$

Hence, $\mathbf{X} \psi_{k}^{\bullet}$ is an eigenvector of $\hat{\mathbf{R}}_{x}$ and the eigenvector $\psi_{k}$ can be obtained as

$$
\psi_{k}=\mathbf{X} \psi_{k}^{\bullet}
$$

requiring far less computations than when diagonalizing $\widehat{\mathbf{R}}_{x}$. From now on, the bullet $(\bullet)$ notation is discarded since it is obvious from the context which of the two correlation matrices is dealt with.

The above assumption of $\mathbf{x}$ being a zero-mean process can hardly be considered valid when the beats $\mathbf{x}_{1}, \ldots, \mathbf{x}_{M}$ originate from one subject and have similar morphology. While it may be tempting to apply PCA on $\mathbf{X}$ once the mean beat has been subtracted from each $\mathbf{x}_{i}$, such an approach would discard important information. The common approach is therefore to apply PCA directly on $\mathbf{X}$, implying that the analysis no longer maximizes the variance in (4), but rather the energy. Figure 2 illustrates PCA for the twodimensional case (i.e., $N=2$ ) when the mean is either unaltered or subtracted.

\subsection{Relationship to the Karhunen-Loève transform}

The KLT is derived as the optimum orthogonal transform for signal representation in terms of the minimum mean square error (MSE) $[11,12]$. Similar to PCA, it is assumed that $\mathbf{x}$ is a random process characterized by the correlation matrix $\mathbf{R}_{x}=$ $E\left[\mathbf{x x}^{T}\right]$. The orthonormal linear transform of $\mathbf{x}$ is obtained by

$$
\mathbf{w}=\Phi^{T} \mathbf{x}
$$

where the set of basis functions $\Phi=\left[\begin{array}{llll}\varphi_{1} & \varphi_{2} & \cdots & \varphi_{N}\end{array}\right]$ is to be determined so that $\mathbf{x}$ can be accurately represented in the minimum MSE sense using a subset of functions and the KLT coefficients $w_{1}, \ldots, w_{K}$. Decomposing $\mathbf{x}$ into a signal estimate $\hat{\mathbf{x}}$, involving the first $K(<N)$ basis functions, and a truncation error $\mathbf{v}$,

$$
\mathbf{x}=\boldsymbol{\Phi} \mathbf{w}=\hat{\mathbf{x}}+\mathbf{v}
$$

with

$$
\begin{gathered}
\widehat{\mathbf{x}}=\sum_{k=1}^{K} w_{k} \boldsymbol{\varphi}_{k}, \\
\mathbf{v}=\sum_{k=K+1}^{N} w_{k} \boldsymbol{\varphi}_{k},
\end{gathered}
$$

the goal is to choose $\Phi$ so that the truncation error $\mathbb{E}=$ $E\left[\mathbf{v}^{T} \mathbf{v}\right]$ is minimized. It can be shown that the optimal set of basis functions is produced by the eigenvector equation for $\mathbf{R}_{x}$,

$$
\mathbf{R}_{x} \boldsymbol{\Phi}=\boldsymbol{\Phi} \Lambda
$$

where the columns of $\boldsymbol{\Phi}$ contain the eigenvectors of $\mathbf{R}_{x}$ and the corresponding eigenvalues $\lambda_{1}, \ldots, \lambda_{N}$ are contained in the diagonal matrix $\Lambda$. The MSE truncation error $\mathcal{E}$ is given by

$$
\mathcal{E}=\sum_{k=K+1}^{N} \lambda_{k}
$$

which is minimized when the $N-K$ smallest eigenvalues 


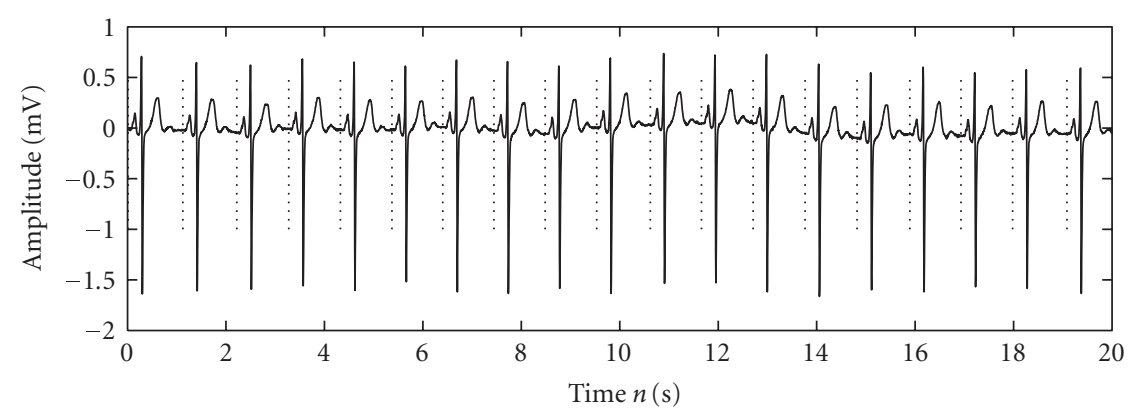

(a)
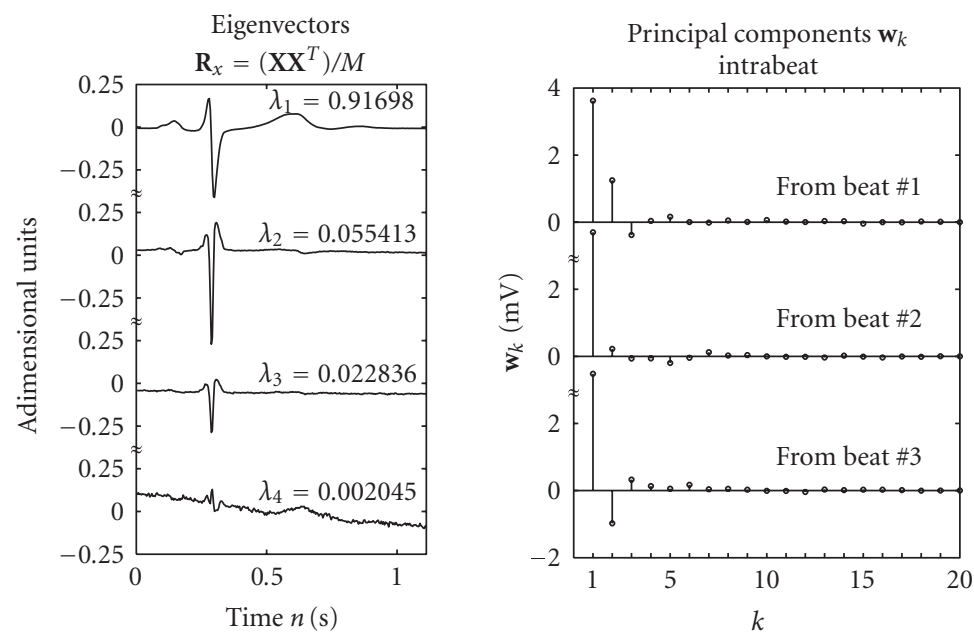

(b)
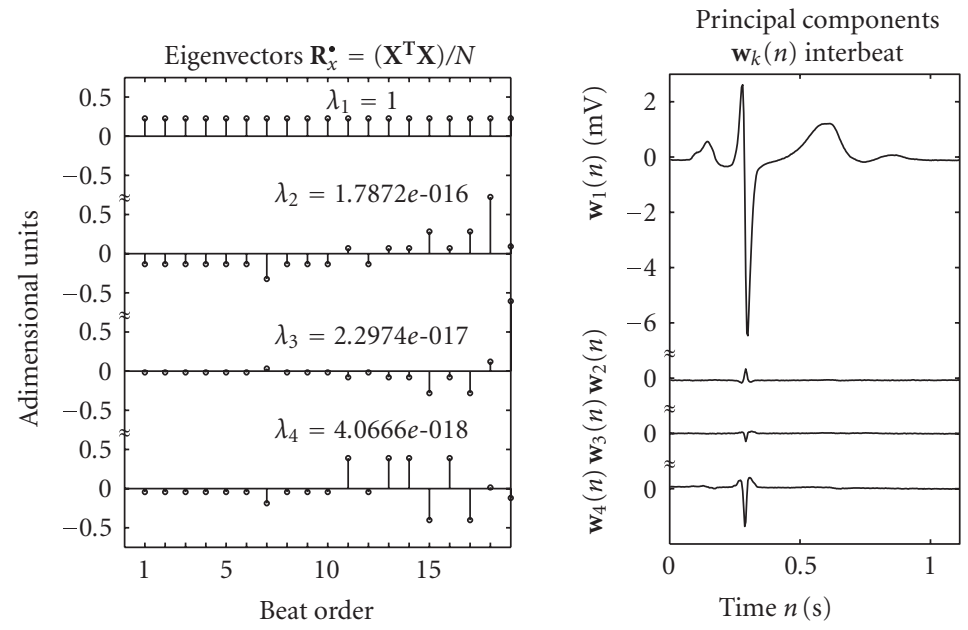

(c)

FIGURE 1: Transform-based representation of an ECG signal (a) segmented to include the whole beat (vertical lines) and produce the data matrix X. The eigenvectors (apart from a DC level) and principal components are displayed for $\hat{\mathbf{R}}_{x}$ obtained as (b) the intrabeat correlation matrix defined in (7), or (c) the interbeat correlation matrix defined in (9).

are chosen since the sum of the eigenvalues then reaches its minimum value. This choice leads to that the eigenvectors corresponding to the $K$ largest eigenvalues should be used as basis functions in (17) in order to achieve the optimal repre- sentation property. From this result, it can be concluded that the PCA and KLT produce identical results as they both make use of the eigenvectors of $\mathbf{R}_{x}$ to transform $\mathbf{x}$ into the principal components/KLT coefficients $\mathbf{w}$, that is, $\boldsymbol{\Psi} \equiv \boldsymbol{\Phi}$. 


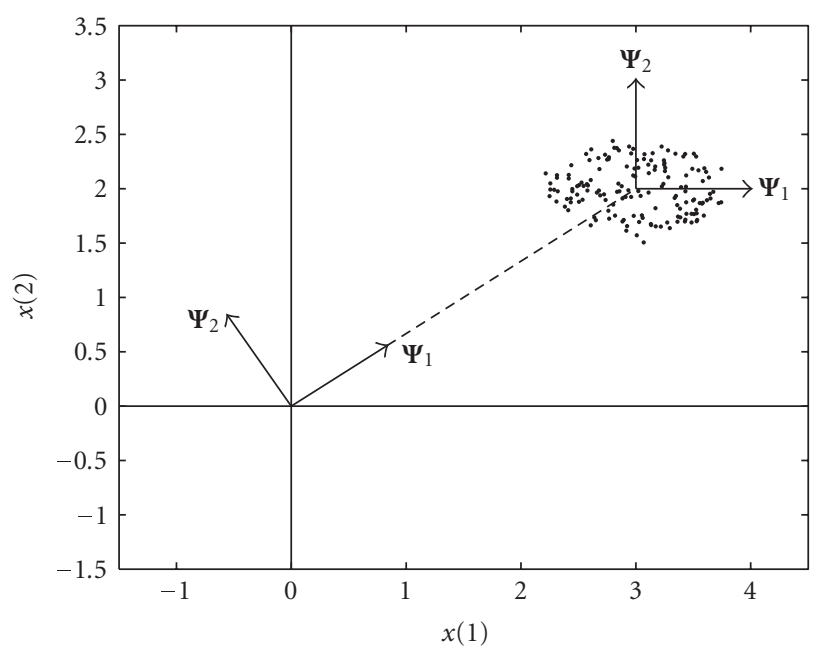

FIgURE 2: Eigenvectors $\psi_{1}$ and $\psi_{2}$ for $N=2$, representing the directions to which the data should be projected (transformed) in order to produce the principal components of the displayed data set. Eigenvectors with origin at $\left[\begin{array}{ll}0 & 0\end{array}\right]$ result from non-zero mean data, whereas eigenvectors with origin at the gravity center of the data result from data when mean is subtracted; note that either variance or energy is maximized depending on the case considered.

\subsection{Singular value decomposition}

The eigenvectors associated with PCA or the KLT can also be determined directly from the data matrix $\mathbf{X}$ using SVD, rather than from $\mathbf{R}_{x}$. The SVD states that an $N \times M$ matrix can be decomposed as [13]

$$
\mathbf{X}=\mathbf{U} \Sigma \mathbf{V}^{T},
$$

where $\mathbf{U}$ is an $N \times N$ orthonormal matrix whose columns are the left singular vectors, and $\mathbf{V}$ an $M \times M$ orthonormal matrix whose columns are the right singular vectors. The matrix $\Sigma$ is an $N \times M$ nonnegative diagonal matrix containing the singular values $\sigma_{1}, \ldots, \sigma_{N}$,

$$
\boldsymbol{\Sigma}=\left[\begin{array}{cccccc}
\sigma_{1} & 0 & \cdots & 0 & \cdots & 0 \\
0 & \sigma_{2} & \cdots & 0 & \cdots & 0 \\
\vdots & \vdots & \ddots & \vdots & \ddots & \vdots \\
0 & 0 & \cdots & \sigma_{N} & \cdots & 0
\end{array}\right],
$$

assuming that $N<M$.

Using the SVD, the sample correlation matrix $\hat{\mathbf{R}}_{x}$ in (7) can be expressed in terms of $\mathbf{U}$ and a diagonal matrix $\boldsymbol{\Lambda}$ whose entries are the normalized and squared singular values $\sigma_{1}^{2} / M, \ldots, \sigma_{M}^{2} / M$,

$$
\hat{\mathbf{R}}_{x}=\frac{1}{M} \mathbf{X} \mathbf{X}^{T}=\frac{1}{M} \mathbf{U} \mathbf{\Sigma} \mathbf{V}^{T} \mathbf{V} \boldsymbol{\Sigma}^{T} \mathbf{U}^{T}=\mathbf{U} \mathbf{\Lambda} \mathbf{U}^{T}
$$

Comparing (23) with (6) and (19), it is obvious that the eigenvectors associated with PCA and the KLT are obtained as the left singular vectors of $\mathbf{U}$, that is, $\Psi=\mathbf{U}$, and the eigenvalues $\lambda_{k}$ as $\sigma_{k}^{2} / M$. In a similar way, the right singular vectors of $\mathbf{V}$ contain information on interbeat correlation, since they are associated with the sample correlation $\hat{\mathbf{R}}_{x}$ in (9).

\subsection{Multilead analysis}

Since considerable correlation exists between different ECG leads, certain applications such as data compression of multilead ECGs can benefit from exploring interlead information rather than just processing one lead at a time. In this section, the single-lead ECG signal of (1) is extended to the multilead case by introducing the vector $\mathbf{x}_{i, l}$, where the indices $i$ and $l$ denote beat and lead numbers, respectively. The $N \times L$ matrix $\mathbf{D}_{i}$ contains all $L$ leads of the $i$ th beat,

$$
\mathbf{D}_{i}=\left[\begin{array}{llll}
\mathbf{x}_{i, 1} & \mathbf{x}_{i, 2} & \cdots & \mathbf{x}_{i, L}
\end{array}\right]
$$

\subsubsection{Lead piling}

A straightforward approach to applying PCA/KLT on multilead ECGs is to pile up the leads $\mathbf{x}_{i, 1}, \ldots, \mathbf{x}_{i, L}$ of the $i$ th beat into an $L N \times 1$ vector $\mathbf{x}_{i}^{\prime}$, defined by

$$
\mathbf{x}_{i}^{\prime}=\left[\begin{array}{c}
\mathbf{x}_{i, 1} \\
\mathbf{x}_{i, 2} \\
\vdots \\
\mathbf{x}_{i, L}
\end{array}\right]
$$

The piling operation is illustrated in Figure 3 for the case with $L=2$. Once all beats have been piled up into a set of $M$ vectors, the ensemble of beats is represented by the $L N \times M$ multilead data matrix

$$
\mathbf{X}^{\prime}=\left[\begin{array}{llll}
\mathbf{x}_{1}^{\prime} & \mathbf{x}_{2}^{\prime} & \cdots & \mathbf{x}_{M}^{\prime}
\end{array}\right]
$$

Accordingly, $\mathbf{X}^{\prime}$ replaces $\mathbf{X}$ in the above calculations required for determining the eigenvectors of the sample correlation matrix. Once PCA/KLT has been performed on the piled vector, the resulting eigenvectors are "depiled" so that the desired principal components/KLT coefficients can be determined for each lead.

\subsubsection{Lead correlation}

In certain studies, the SVD is applied directly to the multilead data matrix $\mathbf{D}_{i}$, thus bypassing the above lead piling operation. Similar to the single-lead case above, the related left singular vectors of $\mathbf{U}$ contain temporal information, however, the right singular vectors of $\mathbf{V}$ contain information on interlead correlation (note that this case resembles the abovementioned situation where interbeat correlation was analyzed, cf. (9)). Hence, by considering all $L$ leads at a certain time $n$, represented with the vector

$$
\mathbf{x}_{i}(n)=\left[\begin{array}{c}
x_{i, 1}(n) \\
x_{i, 2}(n) \\
\vdots \\
x_{i, L}(n)
\end{array}\right]
$$




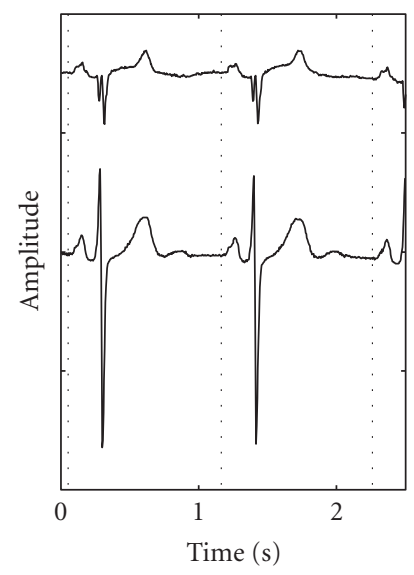

(a)

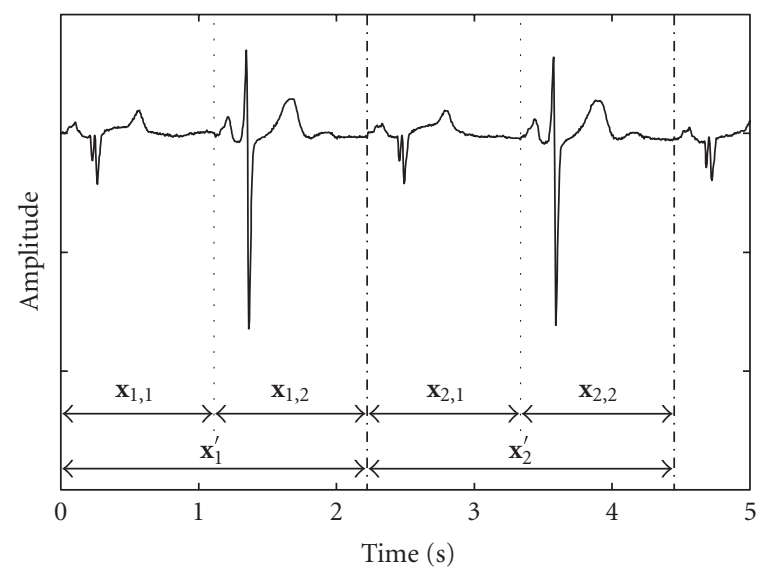

(b)

FIGURE 3: Concatenation of a two-lead ECG signal containing two beats. (a) Each beat of the two leads is piled up into (b) one single vector.

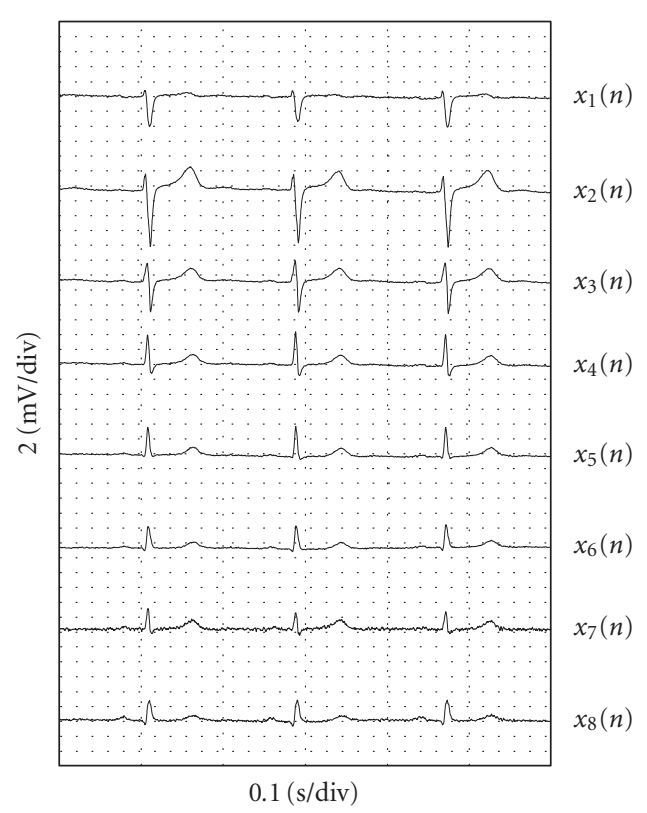

(a) Original ECG

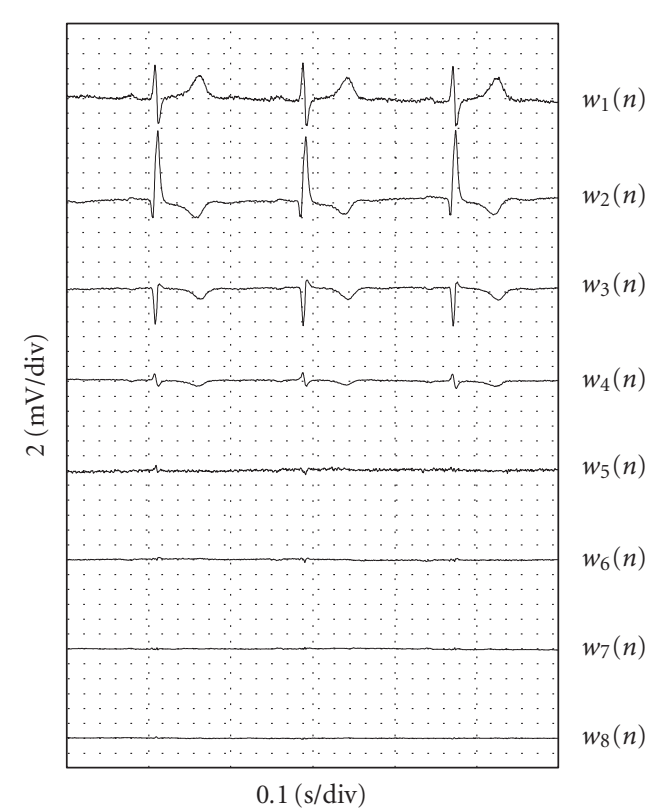

(b) Transformed ECG

FIgURe 4: (a) The standard 12-lead ECG $\left(V_{1}, \ldots, V_{6}, I\right.$, and $I I$ from top to bottom) and (b) its KL transform, obtained using (28), which concentrates the signal energy to only three of the leads.

the PCA/KLT can be used to concentrate the information into fewer leads, using

$$
\mathbf{w}_{i}(n)=\mathbf{V}^{T} \mathbf{x}_{i}(n), \quad n=1, \ldots, N .
$$

This lead-reducing transformation is illustrated by Figure 4 for the standard 12-lead ECG (only 8 leads are unique for this lead system). Using the samples of the displayed signal segment to estimate $\mathbf{R}_{\mathbf{x}}$, it is evident that the energy of the original leads is redistributed so that only 3 out of the 8 transformed leads $\mathbf{w}_{i}(n)$ contain significant energy; the remaining leads mostly account for noise although small residues of ventricular activity can be observed.

\subsubsection{Independent time-lead correlation}

A major disadvantage with the lead piling is that the total number of computations amounts to $O\left(N^{3} L^{3}\right)$ [14]. One approach to reduce complexity is to consider the following series expansion of the data matrix $\mathbf{D}$ :

$$
\mathbf{D}=\sum_{n=1}^{N} \sum_{l=1}^{L} w_{n, l} \mathbf{B}_{n, l}
$$

where $\mathbf{B}_{n, l}$ denotes a two-dimensional basis function, depending on time $n$ and lead $l$. In certain situations, it is 
reasonable to assume that the basis functions are separable and described by rank-one matrices,

$$
\mathbf{B}_{n, l}=\mathbf{t}_{n} \mathbf{s}_{l}^{T}
$$

where the time vector $\mathbf{t}_{n}$ constitutes the $n$th column of the $N \times N$ matrix $\mathbf{T}$, and the lead vector $\mathbf{s}_{l}$ constitutes the $l$ th column of the $L \times L$ matrix $\mathbf{S}$; both $\mathbf{T}$ and $\mathbf{S}$ are assumed to be full rank. Then, the series expansion in (29) can be expressed in matrix form as

$$
\mathbf{D}=\mathbf{T W S}^{T},
$$

where $\mathbf{W}$ is an $N \times L$ matrix formed by the coefficients $w_{n, l}$, determined from $\mathbf{D}$ by

$$
w_{n, l}=\mathbf{t}_{n}^{T} \mathbf{D} \mathbf{s}_{l} .
$$

When the correlation function is separable such that

$$
E\left[x_{i, l_{1}}\left(n_{1}\right) x_{i, l_{2}}\left(n_{2}\right)\right]=r^{t}\left(n_{1}, n_{2}\right) r^{s}\left(l_{1}, l_{2}\right),
$$

the correlation matrix of the piled vector $\mathbf{x}^{\prime}$ can be expressed as a Kronecker product,

$$
\mathbf{R}_{\mathbf{x}^{\prime}}=\mathbf{R}^{t} \otimes \mathbf{R}^{s}
$$

where $\mathbf{R}^{t}$ and $\mathbf{R}^{s}$ characterize the temporal and spatial correlations, respectively. It has been shown that the eigenvectors of $\mathbf{R}_{\mathbf{x}^{\prime}}$ can be computed as the outer product of the eigenvectors of $\mathbf{R}^{t}$ and $\mathbf{R}^{s}$, respectively [15]; these two sets of eigenvectors thus constitute the vectors $\mathbf{t}_{n}$ and $\mathbf{s}_{l}$ which define the rank-one matrices $\mathbf{B}_{n, l}$ in (30). The sample correlation matrices of $\mathbf{R}^{s}$ and $\mathbf{R}^{t}$ are obtained by

$$
\begin{gathered}
\hat{\mathbf{R}}^{s}=\frac{1}{M N} \sum_{i=1}^{M} \sum_{n=1}^{N} \mathbf{x}_{i}(n) \mathbf{x}_{i}(n)^{T} \\
\hat{\mathbf{R}}^{t}=\frac{1}{M L} \sum_{i=1}^{M} \sum_{l=1}^{L} \mathbf{x}_{i, l} \mathbf{x}_{i, l}^{T}
\end{gathered}
$$

respectively. With the assumption of a separable correlation function, the computational complexity is reduced from $O\left(N^{3} L^{3}\right)$ to $O\left(N^{3}+L^{3}\right)$.

\subsection{Adaptive coefficient estimation}

In certain applications, truncation of the series expansion into $K$ basis functions, (cf. (17)), is employed for the purpose of improving the signal-to-noise ratio (SNR). Interestingly, the SNR can be further improved when the signal is recurrent since the basis function representation can be combined with adaptive filtering techniques. Such techniques make it possible to track time-varying changes in beat morphology even at relatively low SNRs. The main approaches to adaptive coefficient estimation are the following.

(i) the instantaneous least mean square (LMS) algorithm with deterministic reference input. The coefficients are adapted at every time instant, producing a vector $\mathbf{w}(n)[16-$ 20]; (ii) the block LMS algorithm. The coefficients are adapted only once for each beat "block," producing a vector $\mathbf{w}_{i}$ that corresponds to the $i$ th beat [21].

Although the instantaneous LMS algorithm is the adaptive technique that has received most attention in biomedical signal processing, the block LMS algorithm represents a natural extension of the above series expansion truncation, and is therefore briefly considered below. This algorithm can be viewed as a marriage of single-beat analysis, relying on the inner product computation to obtain the KLT coefficients, and the conventional LMS algorithm. In addition, the block LMS algorithm offers certain theoretical advantages over the instantaneous LMS algorithm with respect to bias and excess MSE (i.e., the error due to fluctuations in coefficient adaptation that cause the minimum MSE to increase).

The derivation of the block LMS algorithm takes its starting point in the MSE criterion, defined by

$$
\mathcal{E}_{\mathbf{w}_{i-1}}=E\left[\left(\mathbf{x}_{i}-\boldsymbol{\Phi}_{s} \mathbf{w}_{i-1}\right)^{T}\left(\mathbf{x}_{i}-\boldsymbol{\Phi}_{s} \mathbf{w}_{i-1}\right)\right],
$$

where the basis function matrix $\Phi$ has been partitioned into signal and noise subspaces,

$$
\Phi=\left[\begin{array}{ll}
\Phi_{s} & \Phi_{v}
\end{array}\right]
$$

The block LMS algorithm iteratively finds the coefficient vector by making use of the steepest descent algorithm [21],

$$
\mathbf{w}_{i}=\mathbf{w}_{i-1}-\frac{1}{2} \mu \nabla_{\mathbf{w}_{i-1}} \boldsymbol{E}_{\mathbf{w}_{i-1}},
$$

where $\mu$ denotes the step size. Following substitution of the gradient expression and replacement of the expected value with its instantaneous estimate, the block LMS algorithm is given by

$$
\mathbf{w}_{i}=(1-\mu) \mathbf{w}_{i-1}+\mu \Phi_{s}^{T} \mathbf{x}_{i}
$$

The algorithm is initialized by $\mathbf{w}_{0}=\mathbf{0}$ which seems to be a natural choice since, apart from $\mu$, it leads to the estimator of $\mathbf{w}_{1}$, that is, $\mathbf{w}_{1}=\mu \boldsymbol{\Phi}_{s}^{T} \mathbf{x}_{1}$. However, initialization to the inner product of the first beat, that is, $\mathbf{w}_{0}=\boldsymbol{\Phi}_{s}^{T} \mathbf{x}_{1}$, reduces the initial convergence time since $\mathbf{w}_{1}=\boldsymbol{\Phi}_{s}^{T} \mathbf{x}_{1}$ [23]. The block LMS algorithm remains stable for $0<\mu<2$.

The block LMS algorithm reduces to single-beat analysis when $\mu=1$, since (39) then becomes identical to (15). When a complete series expansion is considered, that is, $K=N$, the block LMS algorithm becomes identical to conventional exponential averaging. However, for the case of most practical interest, that is, $K<N$, the block LMS algorithm performs exponential averaging of the coefficient vector: an operation which produces a less noisy estimate of the coefficient vector, but also less capable of tracking dynamic signal changes.

For the steady-state condition when $\mathbf{x}_{i}$ is composed of a fixed signal component $s$ and a time-varying noise component $\mathbf{v}_{i}$, the block LMS algorithm can, in contrast to the instantaneous LMS algorithm, be shown to produce a steadystate coefficient vector $\mathbf{w}_{\infty}$ which is an unbiased estimate of the optimal MSE solution [21]. Another attractive property 


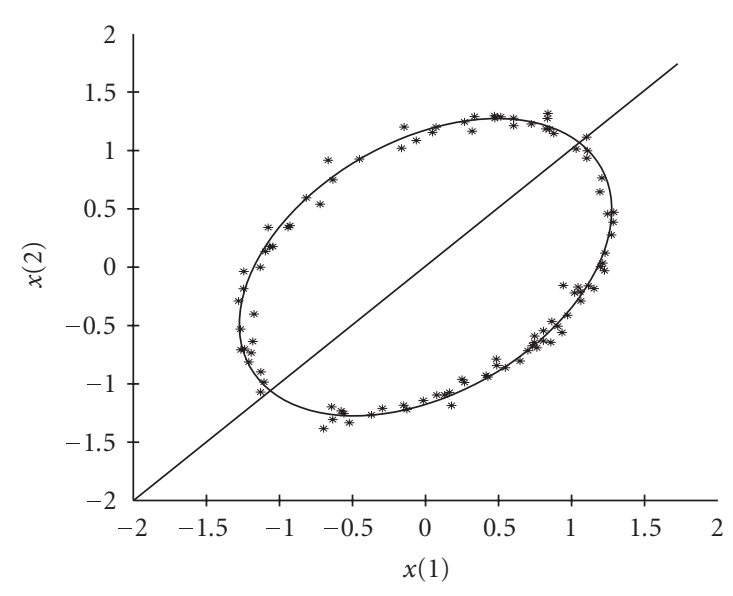

(a)

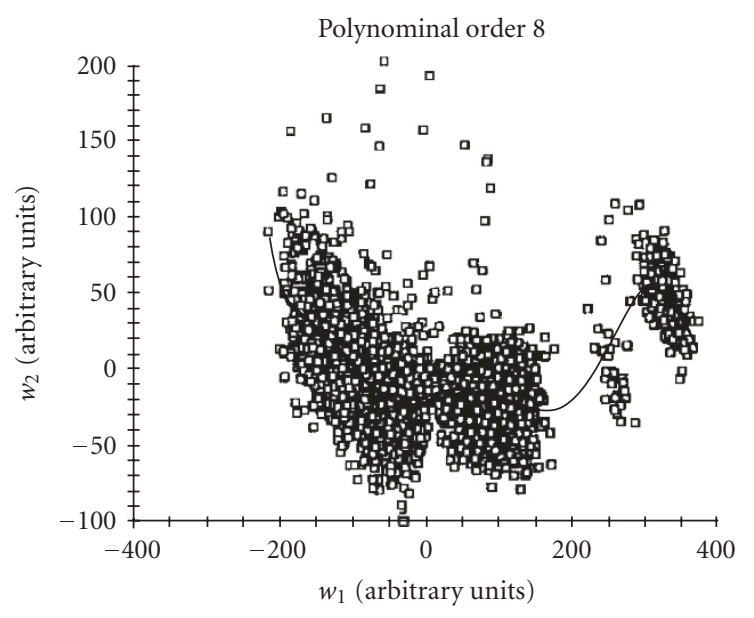

(b)

Figure 5: (a) Plot of the two-sample data $x(1)$ and $x(2)$ generated by the hidden factor $\phi$, see text, with Gaussian white noise added. The straight line represents the first eigenvector that results from PCA, whereas the circle represents the parametric curve that results from nonlinear PCA. It is clear that the projection error on the straight line is much larger than on the elliptic curve, and therefore nonlinear PCA has a superior concentration capability in this particular example. (b) An example of a nonlinear function (polynomial) capturing the dependency between the two largest principal components. (Reprinted from [22] with permission.)

of the block LMS algorithm is that its excess MSE is given by

$$
\mathcal{E}_{\mathrm{ex}}(\infty)=\frac{\mu K}{(2-\mu) N} \sigma_{v}^{2}
$$

where $\sigma_{v}^{2}$ denotes the variance of the noise component. This expression does not involve any term due to the truncation error as does the excess MSE for the instantaneous LMS algorithm, and therefore, the block LMS algorithm is always associated with a lower excess MSE [10]. This property becomes particularly advantageous when the signal energy is concentrated to a few basis functions.

\subsection{Nonlinear principal component analysis}

In certain situations, it is possible to further concentrate the variance of the principal components using a nonlinear transformation, making the signal representation even more compact than with linear PCA. This property can be illustrated by the two-sample data vector $\mathbf{x}=\left[\begin{array}{lll}x(1) & x(2)\end{array}\right]^{T}=$ $[\cos (\phi) \sin (\phi)]^{T}$, being completely defined by the uniformly distributed angle $\phi$ [24]. Applying PCA to samples resulting from different outcomes of $\phi$, it is evident that the first principal component does not approximate the data adequately, see Figure 5(a). The parametric curve determined by the "hidden" factor $\phi$, nonlinearly related to the samples through $\phi=h(\mathbf{x})=\cos ^{-1}(x(1))$, produces a much better approximation. It is evident from Figure 5(a) that the use of $\phi$ contributes to a lower error since the error between the ellipsoid and the data is much smaller than the error with respect to the straight line. Using ECG data, Figure 5(b) presents an example in which a nonlinear function (polynomial) captures the relations between the two largest principal components. In this case, the nonlinear, polynomial, relation is shown in the PCA domain rather than in the data domain, but equivalent relations could be displayed in the data domain.

In general, it is assumed that the signal $\mathbf{x}=\left[\begin{array}{lll}x(1) & \cdots\end{array}\right.$ $x(N)]^{T}$ is generated by some underlying feature vector $\phi=$ $\left[\begin{array}{lll}\phi_{1} & \cdots & \phi_{K}\end{array}\right]^{T}, K \leq N$, through $N$ nonlinear continuous decoding functions from $\mathcal{R}^{K}$ to $\mathcal{R}, x(1)=g_{1}(\phi), \ldots, x(N)=$ $g_{N}(\phi)$, which have the inverse coding functions from $\mathcal{R}^{N}$ to $\mathcal{R}, \phi_{1}=h_{1}(\mathbf{x}), \ldots, \phi_{K}=h_{K}(\mathbf{x})$. The coding function $\mathbf{h}=\left[\begin{array}{lll}h_{1} & \cdots & h_{K}\end{array}\right]^{T}$ from $\mathcal{R}^{N}$ to $\mathcal{R}^{K}$ and the decoding function $\mathbf{g}=\left[\begin{array}{lll}g_{1} & \cdots & g_{N}\end{array}\right]^{T}$ from $\mathcal{R}^{K}$ to $\mathcal{R}^{N}$ are members of some sets $\mathcal{F}_{c}$ and $\mathcal{F}_{d}$ of nonlinear functions, respectively. The goal of nonlinear PCA (NLPCA) is to minimize the nonlinear reconstruction mean square error

$$
\varepsilon=E\left[(\mathbf{x}-\mathbf{g}(\mathbf{h}(\mathbf{x})))^{2}\right]
$$

for an optimum choice of $\mathbf{g}$ and $\mathbf{h}$ in the sets $\mathscr{F}_{c}$ and $\mathcal{F}_{d}$, respectively. The solution will depend on the choice of $\mathscr{F}_{c}$ and $\mathcal{F}_{d}$ as well as the signal $\mathbf{x}$. Linear PCA represents a particular case of NLPCA in which the two spaces are related to each other through linear mapping.

Unfortunately, there are in general an infinite number of solutions to the NLPCA minimization problem so that the hidden parameters are not unique. In fact, if a pair of functions, $\mathbf{h}_{1}(\cdot)$ and $\mathbf{g}_{1}(\cdot)$, achieves the minimum error, so does any pair $\mathbf{h}_{1}\left(q^{-1}(\cdot)\right), q\left(\mathbf{g}_{1}(\cdot)\right)$ for any invertible function $q(\cdot)$. However, by keeping either $\mathbf{g}$ or $\mathbf{h}$ fixed, a set can be determined which gives a unique result $[24,25]$ :

(i) the set $\mathscr{F}_{d}=\left\{\mathbf{l}(\phi)\right.$ for all $\left.\phi \in \mathcal{R}^{K}\right\}$ of contours $\mathbf{l}(\phi)=$ $\{\mathbf{x}: \mathbf{h}(\mathbf{x})=\phi\}$ for the function $\mathbf{h}$;

(ii) the set $\mathcal{F}_{c}$ is constituted by the $K$-parametric surface $C=\left\{\mathbf{g}(\phi)\right.$ for all $\left.\phi \in \mathcal{R}^{K}\right\}$ generated by $\mathbf{g}$.

Here, $C$ denotes the so-called $K$-parametric nonlinear principal component surface of $\mathbf{x}$, which in Figure 5 is repre- 
sented by the surface curve from $\mathcal{R}^{1}$ to $\mathcal{R}^{2}$, that is, $\mathbf{x}=$ $\mathbf{g}(\phi)=\left[\cos \left(\phi_{0}\right) \sin \left(\phi_{0}\right)\right]^{T}$.

\section{DATA COMPRESSION}

Since a wide range of clinical examinations involves ECG signals, huge amounts of data are produced not only for immediate scrutiny, but also for database storage for future retrieval and review. Although hard disk technology has undergone dramatic improvements in recent years, increased disk size is parallelled by the ever-increasing wish of physicians to store more information. In particular, the inclusion of additional ECG leads, the use of higher sampling rates and finer amplitude resolution, the inclusion of noncardiac signals such as blood pressure and respiration, and so on, lead to rapidly increasing demands on disk size. An important driving force behind the development of methods for data compression is the transmission of ECG signals across public telephone networks, cellular networks, intrahospital networks, and wireless communication systems. Transmission of uncompressed data is today too slow, making it incompatible with real-time demands that often accompany many ECG applications.

\subsection{Single-lead compression}

Transform-based data compression assumes that a more compact signal representation exists than that of the timedomain samples which packs the energy into a few coefficients $w_{1}, \ldots, w_{K}$. These $K$ coefficients are retained for storage or transmission while the remaining coefficients are discarded as they are near zero. Transform-based compression is usually lossy since the reconstructed signal is allowed to differ from the original signal, that is, the truncation error $\mathbf{v}$ is not retained. Although a certain amount of distortion can be accepted in the reconstructed signal, it is absolutely essential that the distortion remains small enough in order not to alter the diagnostic content of the ECG. Several different sets of basis functions have been investigated for ECG compression purposes, and the KLT is one of the most popular as it minimizes the MSE of approximation [26-32].

Transform-based compression requires that the ECG first be partitioned into a series of successive blocks, where each block is subjected to data compression. The signal may be partitioned so that each block contains one beat. Each block is positioned around the QRS complex, starting at a fixed distance before the QRS, including the $\mathrm{P}$ wave and extending beyond the end of the $\mathrm{T}$ wave to the beginning of the next beat. Since the heart rate varies, the distance by which the block extends after the QRS complex is adapted to the prevailing heart rate. Hence, the resulting blocks vary in length, introducing a potential problem in transform-based compression where a fixed block length is assumed. This problem may be solved by padding too short blocks with a suitable sample value, whereas too long blocks can be truncated to the desired length. The use of variable block lengths has been studied in detail in $[33,34]$; the results show that variable block lengths produce better compression performance than fixed blocks. It should be noted that partitioning of the ECG is bound to fail when certain chaotic arrhythmias are encountered such as ventricular fibrillation during which no QRS complexes are present.

A fixed number of KL basis functions are often considered for data compression, where the choice of $K$ may be based on considerations related to overall performance expressed in terms of compression ratio and reconstruction error. The performance of the KLT can be described by the index $\mathcal{R}_{k}$, defined in (8), which reflects how well the original signal is approximated by the basis functions. While this index describes the performance on the chosen ensemble of data as an average, it does not provide information on the reconstruction error in individual beats. Therefore, it may be appropriate to include a criterion for quality control when $K$ is chosen. Since the loss of morphologic detail causes incorrect interpretation of the ECG, the choice of $K$ can be adapted for every beat to the properties of the reconstruction error $(\mathbf{x}-\widehat{\mathbf{x}})$, where the estimate $\widehat{\mathbf{x}}$ is determined from the $K$ most significant basis function [32], (cf. (17)). The value of $K$ may be chosen such that the root mean square (RMS) value of the reconstruction error does not exceed the error tolerance $\varepsilon$, whereas a more demanding approach is to choose $K$ such that none of the reconstruction errors of the entire block exceeds $\varepsilon$. Yet another approach to the choice of $K$ may be to employ an "analysis-by-synthesis" algorithm which is designed to ensure that the errors in ECG amplitudes and durations do not become clinically unacceptable $[35,36]$.

By letting $K$ be variable, one can fully control the quality of the reconstructed signal, however, one is also forced to increase the amount of side information since the value of $K$ must be stored for every data block. If the basis functions are a priori unknown, a larger number of basis functions must also be part of the side information. Figure 6 illustrates signal reconstruction for a fixed number of basis functions and a number determined by an RMS-based quality control criterion. In this example, the indicated error tolerance is attained by using different numbers of basis functions for each of the three displayed beats.

The estimation of $\mathbf{R}_{x}$ can be based on different types of data sets. The basis functions are labeled "universal" when the data set originates from a large number of patients, and "subject-specific" when the data set originates from a single recording. While it is rarely necessary to store or transmit universal basis functions, subject-specific functions need to be part of the side information. Still, subject-specific basis functions offer superior energy concentration of the signal because these functions are better tailored to the data, provided that the ECG contains few beat morphologies. Figure 7 illustrates the latter observation by presenting the reconstructed signal for both types of basis functions. One approach to reduce the side information is to employ wavelet packets since these approximate to the KLT by efficiently coding the basis functions [37].

A limitation of the KL basis functions comes to light when compressing ECGs with considerable changes in heart rate and, consequently, changes in the position of the $\mathrm{T}$ wave. Such ECG changes are observed, for example, during the 


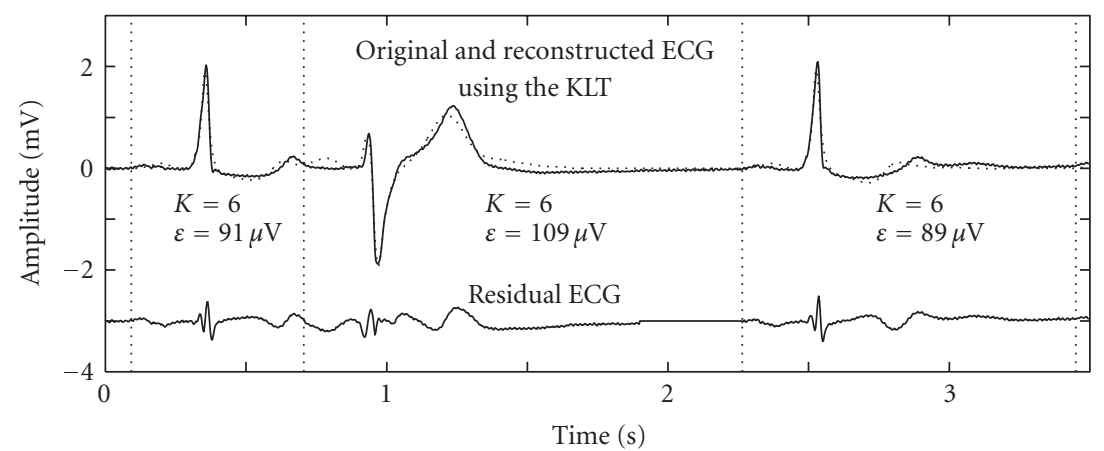

(a)

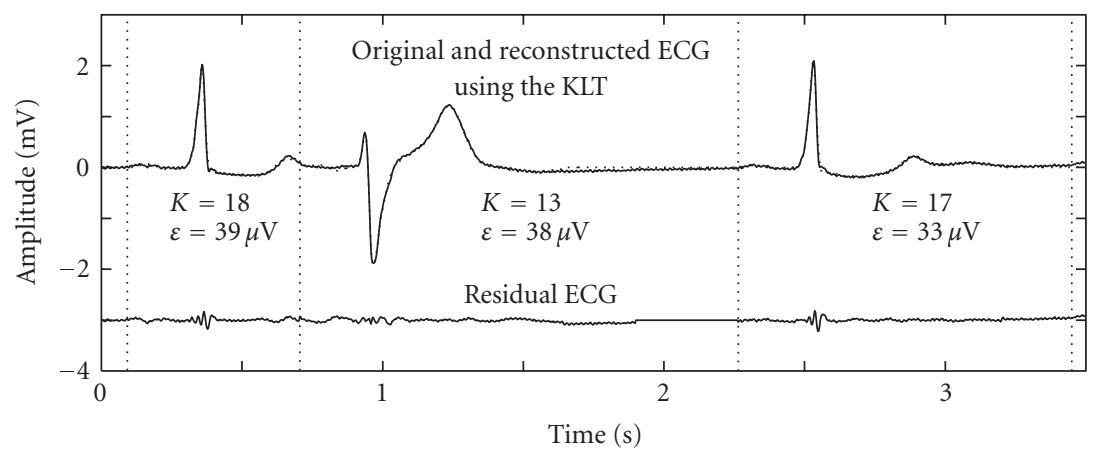

(b)

Figure 6: Quality control and KLT-based data compression. (a) A fixed number of basis functions $(K=6)$ is used for signal reconstruction. (b) The number of basis functions is chosen so that the RMS error, denoted as $\varepsilon$, between the original and reconstructed signals is always below $40 \mu \mathrm{V}$. For ease of interpretation, the residual ECG is plotted with a displacement of $-3 \mathrm{mV}$.

course of a stress test. Since the basis functions account for the $\mathrm{T}$ wave occurrence at a fixed distance from the QRS complex, the basis functions become ill-suited for representing beats whose $\mathrm{T}$ waves occur earlier or later than this interval. As a result, additional basis functions are required to achieve the desired reconstruction error, thus leading to less efficient compression. The representation efficiency can be improved by resampling of the ST-T segment in relation to the length of the preceding RR interval.

A nonlinear variant of PCA has also been considered for single-lead data compression, the goal being to exploit the nonlinear relationship between different principal components [22]. The method is based on the assumption that higher-order components can be estimated from knowledge of the first $k$ components by $\hat{w}_{i}=f_{i, k}\left(w_{1}, \ldots, w_{k}\right), i>k$, without having to store the higher-order components ( $k$ was set to 1 in [22]). Although the coefficients that define the nonlinear functions must be stored, their storage requires very few bytes. One way to model the nonlinear relationship is to use a polynomial with a small number of coefficients (typically 7 or 8$)$, see Figure $5(\mathrm{~b})$.

\subsection{Multilead compression}

With transform-based methods, interlead correlation may be dealt with in two steps, namely, a transformation which concentrates the signal energy spread over the available $L$ leads into a few leads, followed by compression of each transformed lead using a single-lead technique. Following con- centration of the signal energy using the transform in (28), different approaches to data compression may be applied to the transformed leads, of which the simplest one is to just retain those leads whose energy exceeds a certain limit. Each retained lead is then compressed using the above single-lead methods, or some other compression techniques. If a more faithful reconstruction of the ECG is required, leads with less energy can be retained, although they will be subjected to more drastic compression than the other leads [38].

A unified approach, which jointly deals with intersample and interlead redundancy, is to pile all segmented leads $\mathbf{x}_{i, 1}, \ldots, \mathbf{x}_{i, L}$ into a single vector $\mathbf{x}_{i}^{\prime}$ subjected to compression using any of the single-lead transform-based methods described above [29, 39]. Applying the KLT, lead piling offers a more efficient signal representation than does the twostep approach, although the calculation of basis functions through diagonalization of the $L N \times L N$ correlation matrix is much more costly, in terms of computational measures, than for the $L \times L$ matrix in (9). However, when it is reasonable to assume that the time-lead correlation is separable, (cf. (30)), the computational load can be substantially reduced.

\section{MYOCARDIAL ISCHEMIA}

Myocardial ischemia arises when the blood flow to cardiac cells is reduced, caused by occlusion or narrowing of one or more of the coronary arteries. As a result, the demand for oxygenated blood to the heart muscle increases, especially during exercise or mental stress. A temporary reduction in 

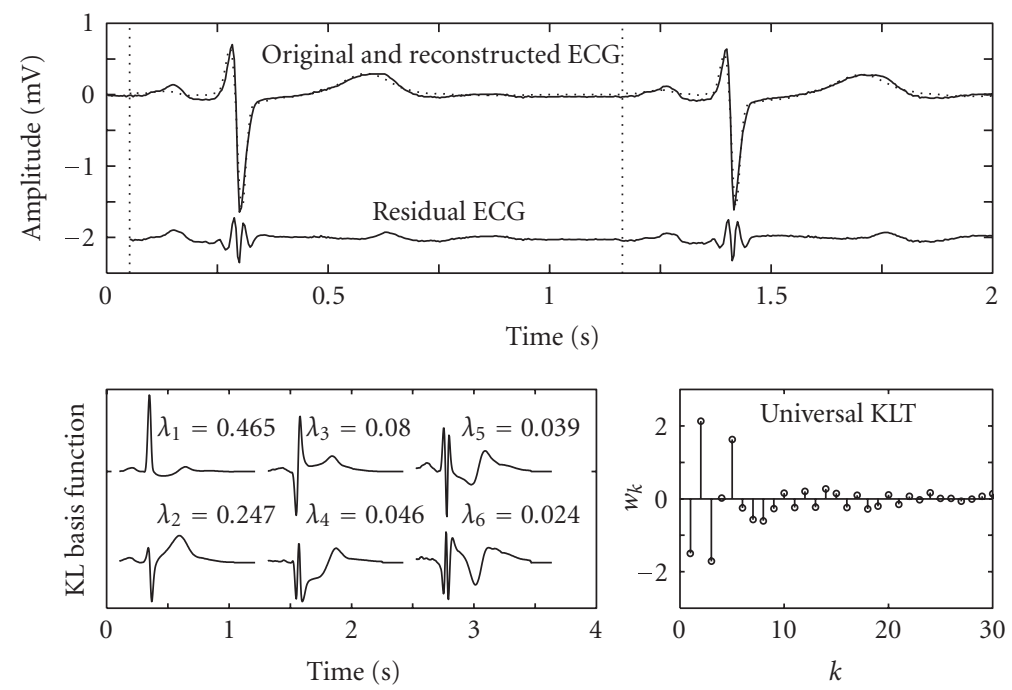

(a)
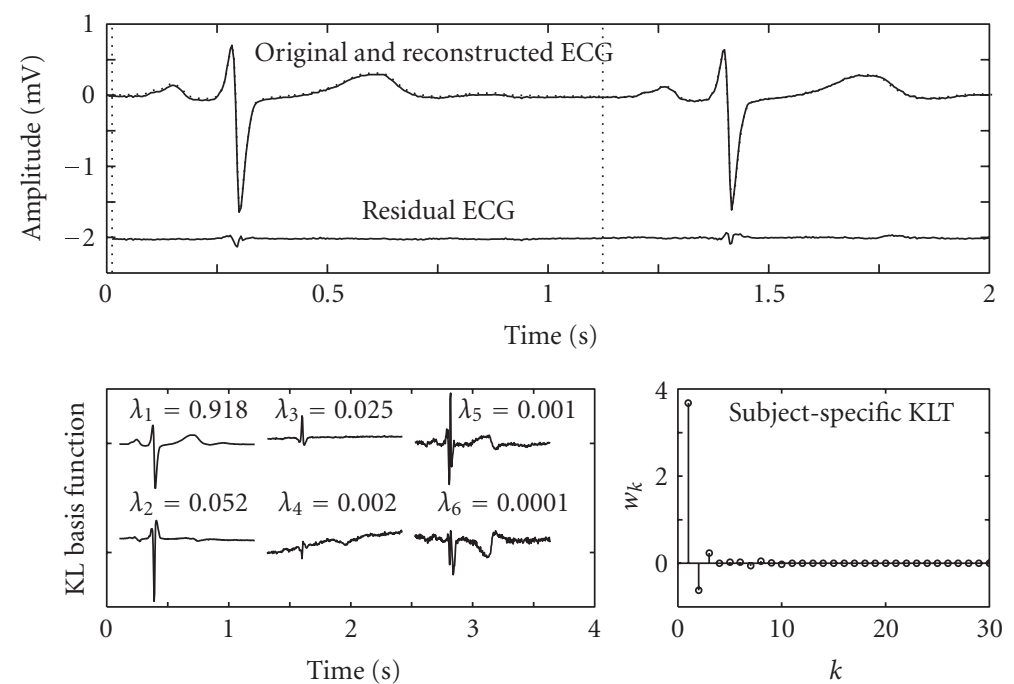

(b)

FIGURE 7: Transform-based data compression using the KL basis functions derived from either (a) a huge database including thousands of ECGs from different subjects or (b) subject-specific data. The basis functions $\varphi_{k}$ and associated eigenvalues $\lambda_{k}$ are presented as are the 30 largest coefficients of the original ECG's KLT. The ECGs are reconstructed with $K=8$ and 2 for universal and subject-specific basis functions, respectively. For ease of interpretation, the residual ECG is plotted with a displacement of $-2 \mathrm{mV}$.

flow often causes chest pain or discomfort (angina pectoris), but can also be completely unrelated to chest pain (silent ischemia). Ischemia is associated with electrical instability of the heart that may initiate life-threatening ventricular tachyarrhythmias such as ventricular fibrillation.

Myocardial ischemia is usually manifested in the ECG as morphologic changes of the ST segment and T wave, jointly referred to as ST-T changes, but may also occur unnoticed. While the normal ST segment starts at the isoelectric line and curves smoothly upwards into the T wave, the ischemic ST segment is instead horizontal or slopes downwards and may start well below the isoelectric line. An ST segment which drops below the isoelectric line is referred to as an ST depression. An ischemic $\mathrm{T}$ wave is often more flat than a normal $\mathrm{T}$ wave and may exhibit biphasic morphology or negative polarity.

Electrocardiographic monitoring during exercise, ambulatory conditions, or in the coronary care unit is essential since it is of interest to detect ischemic ST-T changes. Historically, such monitoring has been based on local amplitude indices, such as the amplitude 60 milliseconds after the $J$ point. However, it is clear that local indices only provide a very limited characterization of the ST-T segment, and therefore, it is important to develop methods which also characterize segment morphology. One such method is based on the assumption that a reduced set of KLT coefficients can be used to detect ischemia [34, 40, 41]; each coefficient reflecting to what degree different segment morphologies are present. 


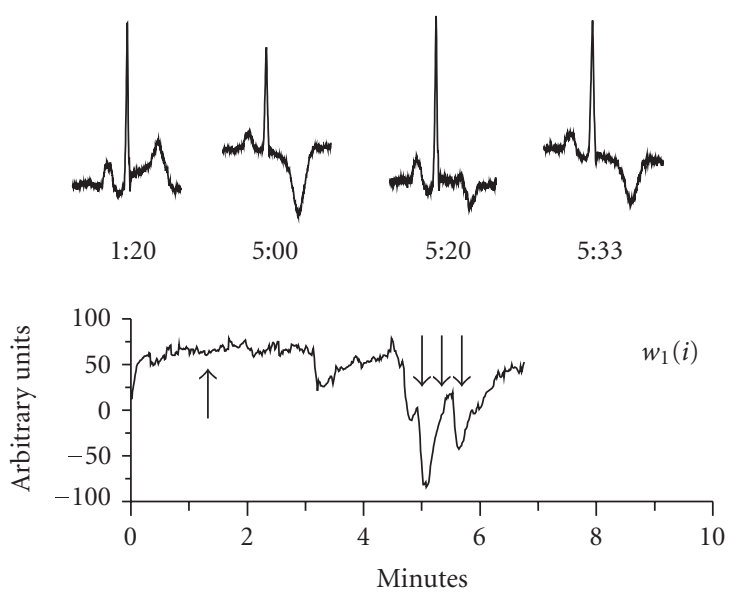

FIgURE 8: The series of the most significant KLT coefficient $w_{1}(i)$ obtained from a patient undergoing balloon inflation in a coronary artery. The four beats (upper panel) correspond to the times indicated by the arrows on the $w_{1}(i)$ series (lower panel). Note that the ST-T segment is initially positive, as reflected by positive values of $w_{1}(i)$, but later changes polarity is inverted and exhibits considerable variation in magnitude.

This method is illustrated by Figure 8 , where the ischemic episode is well-reflected by the drastic changes that occur in the most significant KL coefficient. It has been shown that this method offers better performance, that is, higher sensitivity and specificity, than the classical indices [42].

Using a universal data set of 100000 beats, it has been shown that four KLT coefficients are sufficient to represent $90 \%$ of the ST-T segment energy [34]. Before the correlation matrix of the data set is estimated, it is essential to remove baseline wander so as to not account for that unwanted activity in the analysis. Moreover, it may be necessary to "normalize" the ST-T segment with respect to heart rate to obtain more efficient signal representation; signals recorded at widely different heart rates should be resampled so that the ST-T segments become more comparable. The resampling strategy has been shown to improve the energy representation by $5 \%$ when considering the first two KLT coefficients [34].

Although the estimation of KLT coefficients can be done with (15), it is suitable to use adaptive techniques, (cf. (39)), to better cope with noise when the ECG is recorded during exercise or ambulatory conditions. Such techniques can be employed with advantage since ischemia-induced ST-T segment changes are gradual, taking place over several beats. Thus, a relatively small value of $\mu$ attenuates noise while still allowing ischemic changes to be tracked, see Figure 9. For example, a value of $\mu$ resulting in a convergence time of 2.5 beats improves the SNR of the coefficient series by a factor 10 without affecting the ability to track changes.

Ischemic changes are more easily detected when multiple leads are considered, suggesting that the coefficients of different leads should be jointly analyzed. Therefore, the detection of ischemic episodes is usually based on a series of coefficients that accumulates the information of individual leads, for example, using

$$
w_{i}^{2}=\sum_{l=1}^{L} w_{i, l}^{2} .
$$

Here, squaring prevents that changes in one lead compensate those of another lead with the opposite sign. Thresholding combined with ad hoc criteria are usually applied to the resulting $w_{i}^{2}$ series for the purpose of detecting the occurrence of ischemic episodes $[40,43]$. It should be noted that spatial information, as provided by the different coefficient series' $w_{i, l}$, has also been found valuable for identification of the occluded artery [44].

Unfortunately, ST-T segment changes are not univocal to ischemia, but a change in body position is often manifested as a shift in the electrical axis that may be misclassified as an ischemic event. In fact, body-position-induced changes in ST$\mathrm{T}$ amplitude exceeding $400 \mu \mathrm{V}$ are not uncommon. However, the occurrence of a body position change (BPC) can be distinguished by analyzing the signature of the KLT coefficients of the QRS complex. While the QRS KLT coefficients change considerably during a BPC, these coefficients are much less influenced when an ischemic episode occurs [45].

\section{VENTRICULAR REPOLARIZATION}

The study of temporal and spatial heterogeneities of ventricular repolarization is essential when investigating cardiac abnormalities such as left ventricular hypertrophy, or the long QT syndrome prone to ventricular tachyarrhythmias that may eventually lead to sudden cardiac death [46]. Temporal information on the repolarization has traditionally been synonymous to the QT interval measurement. However, the length of this interval depends on heart rate and must therefore be corrected before evaluating its diagnostic impact. Several correction techniques exist, ranging from simple Bazett's correction [47] to more advanced corrections which account for individual dependencies and preceding RR intervals [48].

Spatial information on repolarization is often quantified by QT dispersion (QTd), measured as the maximum differences between QT intervals measure of available leads [49]. More recently, this measurement has been seriously questioned, suggesting that QTd is more a result of different ST-T loop projections on different leads rather than true dispersion of the repolarization [50]. This limitation, together with the fact that temporal indices only partially can describe repolarization, has spawned various efforts to develop repolarization indices that better characterize the information contained in the ST-T segment. Other characteristics related to T wave morphology may have important clinical implications. These efforts resulted in some PCA-based techniques whose aim is to extract valuable information from the $\mathrm{T}$ wave [8].

The total cosine R-to- $\mathrm{T}$ descriptor $\mathrm{T}_{\mathrm{CRT}}$ is defined as the cosine angle between the dominant vectors of depolarization and repolarization as measured in a three-dimensional loop $[5,51,52]$. The three dominant transformed leads are computed according to (28) to form the dipolar signal $\mathbf{s}_{D}(n)=$ $\left[\begin{array}{ccc}w_{1}(n) & w_{2}(n) & w_{3}(n)\end{array}\right]^{T}$, but where the segmented matrix $\mathbf{D}$ 

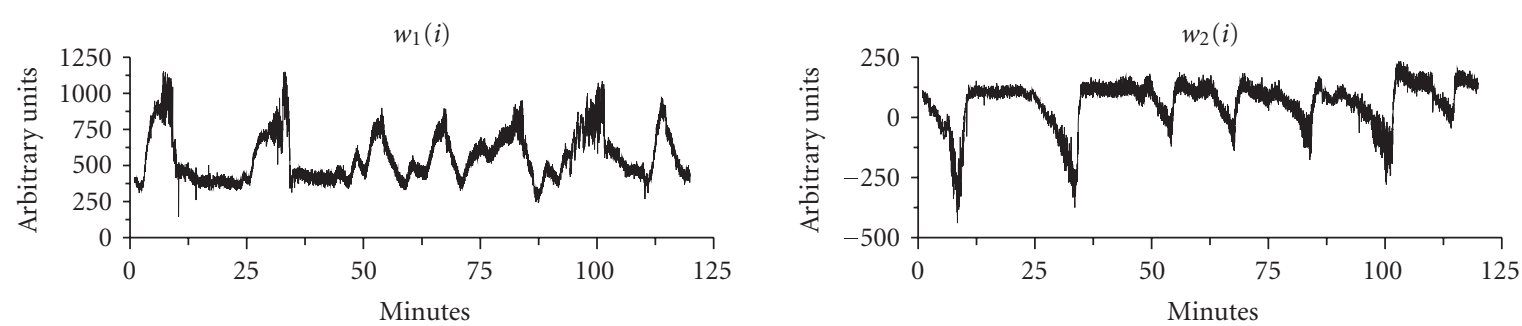

(a)
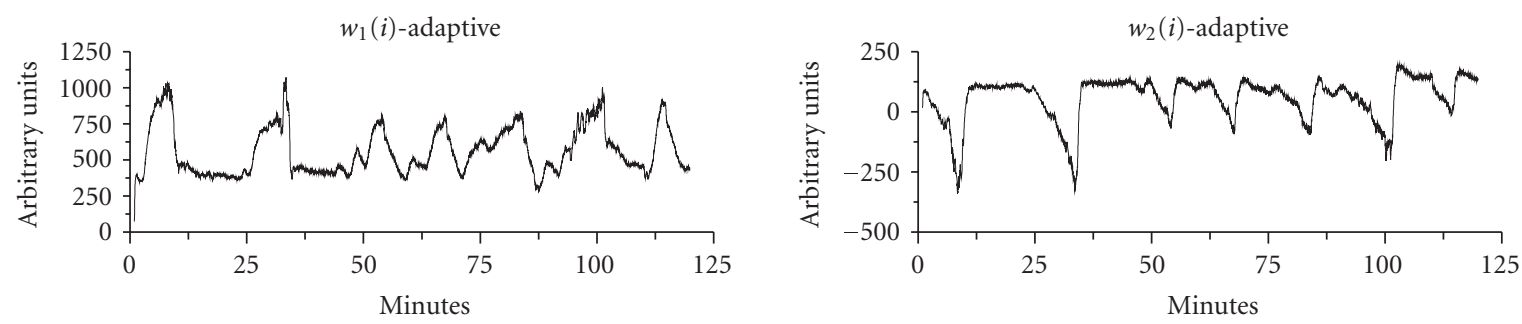

(b)

FIGURE 9: Ischemia and ST-T segment analysis during noisy conditions. The KLT coefficient series' $w_{1}(i)$ and $w_{2}(i)$ are obtained using either (a) the inner product or (b) the inner product combined with adaptive estimation. Note the significant reduction in noise level from (a) to (b), while the information on ischemia episodes remains essentially unchanged.

includes the complete heart beat, that is, the PQRST complex. In addition, the fiducial point of the QRS complex, denoted as $n_{\mathrm{QRS}}$, is assumed to be available from some algorithm for waveform delineation; a QRS interval from $n_{\mathrm{QRS}}^{o}$ to $n_{\mathrm{QRS}}^{e}$ centered around $n_{\mathrm{QRS}}$ and lasting for 30 milliseconds is defined, and the T wave peak position $n_{T}$ is estimated as the position where the module signal $\mathbf{s}_{D}^{T}(n) \mathbf{s}_{D}(n)$ reaches its maximum, restricted to be in the ST-T segment. Then, the index $T_{\mathrm{CRT}}$ is defined by

$$
T_{\mathrm{CRT}}=\frac{1}{n_{\mathrm{QRS}}^{e}-n_{\mathrm{QRS}}^{o}+1} \sum_{n=n_{\mathrm{QRS}}^{o}}^{n_{\mathrm{QRS}}^{o}} \cos \angle\left(\mathbf{s}_{D}(n), \mathbf{s}_{D}\left(n_{T}\right)\right) .
$$

When a comparison of the ventricular gradient is to be made from beats at different stages of the same recording, hypothesizing that only the $\mathrm{T}$ vector changes with repolarization heterogeneity, the gradient can be estimated with respect to a fixed reference called "total angle principal componentto-T" $T_{\mathrm{PT}}$, reducing the uncertainty of estimating the depolarization reference [53]. This reference $\mathbf{u}$ can be taken to be the dominant direction, $\mathbf{u}=\left[\begin{array}{lll}1 & 0 & 0\end{array}\right]^{T}$, of the dipolar decomposition, resulting in

$$
T_{\mathrm{PT}}=\angle\left(\mathbf{u}, \mathbf{s}_{D}\left(n_{T}\right)\right) \text {. }
$$

Another repolarization index is the total morphology dispersion $T_{\mathrm{MD}}$, computed by first recovering the original signals D, (cf. (24)), after truncating the SVD transformation to the dipolar components. This is done by splitting the $\mathbf{V}=\left[\begin{array}{ll}\mathbf{V}_{3} & \mathbf{V}_{L-3}\end{array}\right]$ and obtaining

$$
\widehat{\mathbf{x}}(n)=\mathbf{V}_{3} \mathbf{V}_{3}^{T} \mathbf{x}(n) .
$$

This new signal $\widehat{\mathbf{x}}(n)$ is again processed to produce an SVDdecomposed signal, but now restricted to the ST-T segment, obtaining the transformation matrix V̌ from which we concentrate on the first two transformed leads $\check{\mathbf{V}}_{2}$ assumed to contain the more important information of the ST-T segment. By analyzing the reconstruction equation in (45), now applied to $\check{\mathbf{V}}_{2}, \check{\mathbf{x}}(n)=\check{\mathbf{V}}_{2} \check{\mathbf{V}}_{2}^{T} \widehat{\mathbf{x}}(n)$, it is obvious that the rows of $\check{\mathbf{V}}_{2}=\left[\begin{array}{lll}\phi_{1} & \cdots & \phi_{L}\end{array}\right]^{T}$, with $\phi_{l}(2 \times 1)$ reconstruction vectors which can be interpreted as the direction into which the SVD-transformed signal needs to be projected to recover an estimate of the original lead in $\check{\mathbf{x}}(n)$. The angle between two directions, relative to each pair of leads $l_{1}$ and $l_{2}$, can then be calculated as

$$
\alpha_{l_{1}, l_{2}}=\angle\left(\phi_{l_{1}} \phi_{l_{2}}\right)
$$

thus measuring the difference in shape between leads $l_{1}$ and $l_{2}$ (a small angle implies similar shape). The $T_{\mathrm{MD}}$ index is defined as

$$
T_{\mathrm{MD}}=\frac{1}{L(L-1)} \sum_{\substack{l_{1}, l_{2}=1 \\ l_{1} \neq l_{2}}}^{L} \angle\left(\phi_{l_{1}} \phi_{l_{2}}\right),
$$

which reflects the mean dispersion in the projection of the repolarization ST-T segment. A variant on $T_{\mathrm{MD}}$ has been proposed in which $\phi_{l}$ is multiplied with its corresponding eigenvalue [5]; however, this index is more difficult to interpret in geometrical terms.

Microvolt beat-to-beat alternations in $\mathrm{T}$ wave morphology are related to dispersion of repolarization and are 


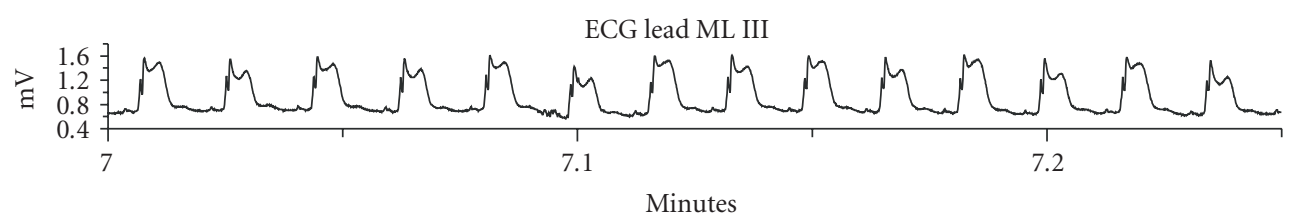

(a)

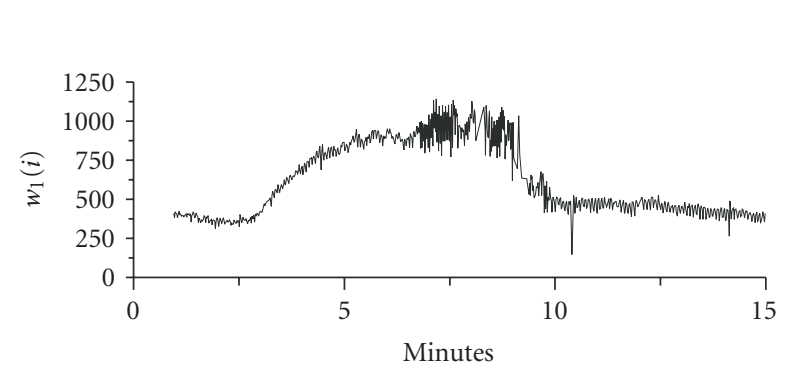

(b)

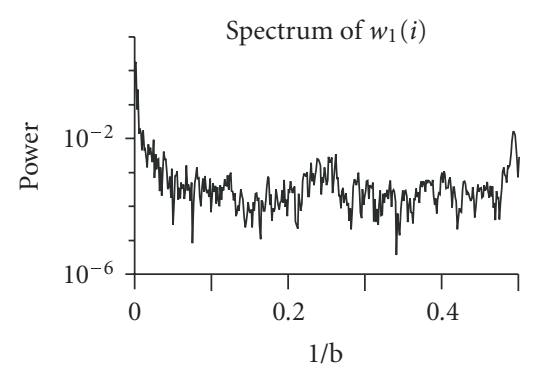

(c)

FIGURE 10: (a) An ECG signal with pronounced T wave alternans during the acute phase of ischemia which has resulted in pronounced ST elevation. (b) The corresponding principal component series $w_{1}(i)$, and (c) the beatquency spectrum. Note the marked peak at 0.5 cycles by beat $(1 / \mathrm{b})$ which reflects the alternans behavior.

considered a presage of malignant ventricular arrhythmias that often lead to sudden cardiac death [54]. The morphologic alternations follow in which every other $\mathrm{T}$ wave has the same morphology. Since most of T wave alternans is a phenomenon in the microvolt range, it cannot be perceived by the naked eye from a standard ECG print-out, but requires signal processing techniques for its detection and quantification [55]. Since the alternans pattern cannot be expected to occur within a well-defined interval of the ST-T segment, it would be helpful to detect and characterize this pattern using a small set of the principal components. Calculation of the principal components from successive beats followed by spectral analysis of the resulting series of principal components is a powerful approach to characterize the oscillatory behavior of the ST-T segment. The alternans pattern can be detected by analyzing the power of the 0.5 beatquency of the series [56], see the example in Figure 10 where only the most significant principal component $w_{1}(i)$ is spectrally analyzed.

\section{ATRIAL FIBRILLATION}

Atrial fibrillation (AF), the most common arrhythmia encountered in clinical practice, has a very complex incompletely understood pathophysiology with various triggers and substrates interacting in multiple ways. Clinically, AF is characterized by progression from paroxysmal to persistent $\mathrm{AF}$, failure to restore and maintain sinus rhythm, but also increased risk of thrombogenesis and embolism [57]. Results of various therapeutic interventions are often disappointing, at least in part due to their empirical application. Subsequently, the search for diagnostic tools to better characterize the disease process in order then to better guide therapeutic decisions has been advocated [58]. It is a common observation that fibrillation waves of the surface ECG have various appearances, ranging from fine to coarse and from disorganized to organized and that the ventricular rate response varies in a rather unpredictable fashion. Even though the first ECG documentation of human AF was made by Einthoven 100 years ago [59], it was just until very recently when ECG analysis of fibrillatory waves was suggested for exploring AF pathophysiology and predicting response to therapy $[60,61]$. The extraction of atrial signals during AF requires advanced signal processing techniques since atrial and ventricular activities overlap in time and frequency, and therefore cannot be separated by linear filtering. Average beat subtraction was initially suggested for atrial signal extraction, relying on the fact that AF is uncoupled to ventricular activity. Hence, subtraction of the average QRST complex produces a residual signal which is the atrial fibrillatory signal [60-62]. More recently, new approaches based on PCA have also been proposed for improved estimation of the atrial signal. In this section we present two different methodologies for analysis of single and multilead recordings $[63,64]$.

\subsection{Single-lead analysis}

A single-lead approach to estimate the atrial signal is valuable for analysis of Holter recordings, being acquired during one or several days. Although modern Holter devices are capable of recording the standard 12-lead ECG, 3-lead devices are commonly employed; even so, it is rather common to record only two leads. Holter recordings are required when it is of interest to monitor paroxysmal AF, that is, when AF initiates and terminates spontaneously, and hence the occurrence of an AF episode is unpredictable.

The spatial information provided by Holter recordings is very limited, thus rendering the multilead PCA less useful. However, the ECG signal also presents a high degree of temporal redundancy which can be exploited in order to cancel the ventricular activity [64]. Indeed, the QRST waveform 


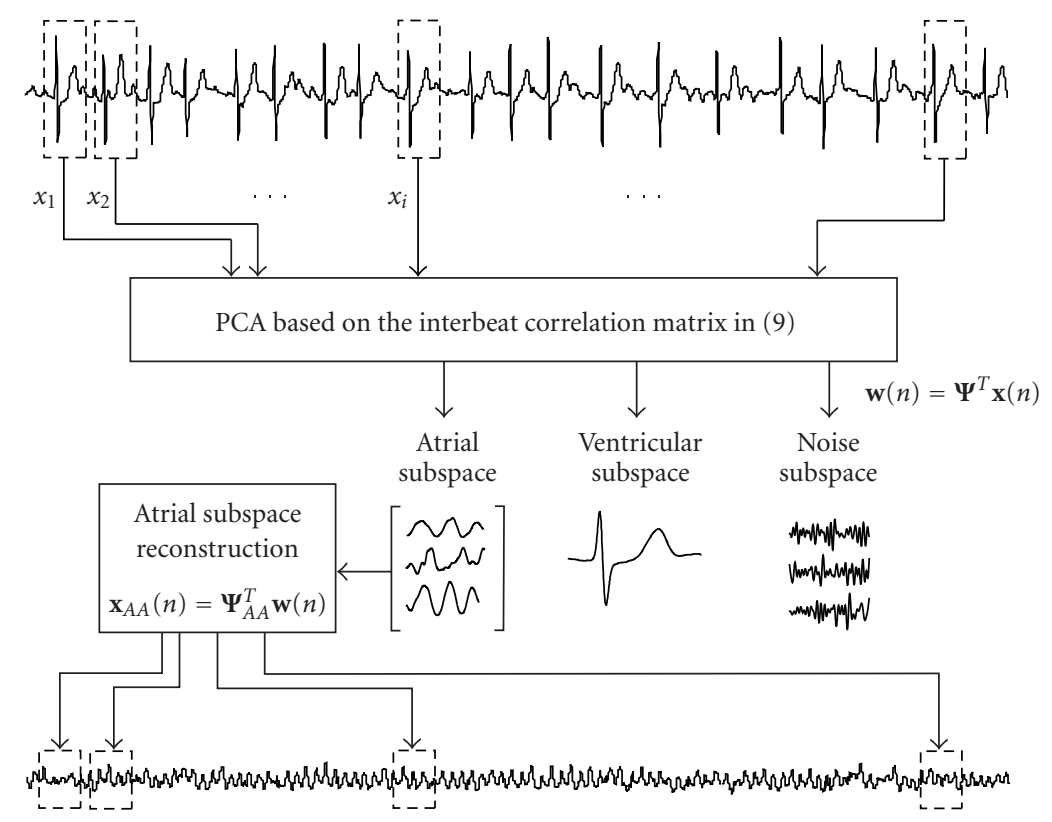

FIGURE 11: Block diagram of interbeat PCA to estimate the atrial activity during AF. The principal components are obtained by (10), and each of the signals in the different subspaces is given by $w_{k}(n)$. The reconstruction is made from the partitioned transformation matrix $\Psi=\left[\Psi_{V A} \Psi_{A A} \Psi_{N}\right]$, generating the reconstructed atrial signal $\mathbf{x}_{A A}(n)$ using $\mathbf{x}_{A A}(n)=\boldsymbol{\Psi}_{A A} \mathbf{w}(n)$ and concatenating back to recover the atrial signal displayed at the bottom.

usually exhibits a recurrent pattern, although different QRST morphologies as well as minor variations in the QRST waveform may occur. For the case when several consecutive beats from the same lead are extracted and PCA is applied to exploit interbeat redundancy, the principal components, ordered according to the eigenvalue sequence, are interpreted as follows.

(1) The most significant component is related to the main QRST waveform. In case of several QRST morphologies, a principal component will represent each of the patterns.

(2) The next few components correspond to dynamics of the QRST waveform. In case of a very regular QRST morphology, these components may be missing.

(3) Subsequently, there are several components related to the atrial activity.

(4) The remaining components correspond to noise.

In addition to the components, PCA outputs the projection of each component has on each beat. Taking these considerations into account, the QRS complex and T wave can be removed at each beat by considering the projections of the ventricular components and removing them from the ECG signal. Equivalently, the same result would be obtained by estimating the atrial activity at each beat from the projections of the nonventricular components (Figure 11).

Cancellation of ventricular activity using the single-lead approach is closely related to adaptive template subtraction, but with the advantage that dynamics in the QRST waveform are also considered, thus producing a more accurate estimate of the atrial signal. This technique has been applied to discriminate nonterminating from terminating AF episodes from Holter recordings [65]. Spectral analysis of the estimated atrial signal revealed that terminating AF had a lower frequency $(3.75-5.5 \mathrm{~Hz})$ than nonterminating AF recordings $(5.5-8 \mathrm{~Hz})$ for the patients under study.

\subsection{Multilead analysis}

The atrial signal can be extracted by exploiting the spatial information in multilead ECGs. By applying PCA to the 12lead ECG, it is possible to remove redundant information contained in the different leads and synthesize them such that the principal components are uncorrelated. Hence, the most representative component is the one which corresponds to the ventricular activity since this activity exhibits the largest energy, whereas the next few components correspond to variability in ventricular activity (cf. the single-lead case above). Among the next principal components, it is possible to find a signal which corresponds to the atrial activity. Figure 12 shows an example where PCA is applied to an AF episode, where the atrial activity can be identified as the fourth principal component, whereas the three first components contain ventricular activity. The detection of the atrial component can be performed using the FFT, since the extracted signal typically exhibits a dominant frequency peak between 3 and $12 \mathrm{~Hz}$. The suitability of PCA for the extraction of the atrial signal has been proposed and validated in [63].

It should be noted that the goal in the AF application is to search for a particular component, instead of maximizing variance or minimizing the MSE. This concept is closely related to blind source separation (BSS) models [66], where the purpose is to estimate a set of independent sources from 


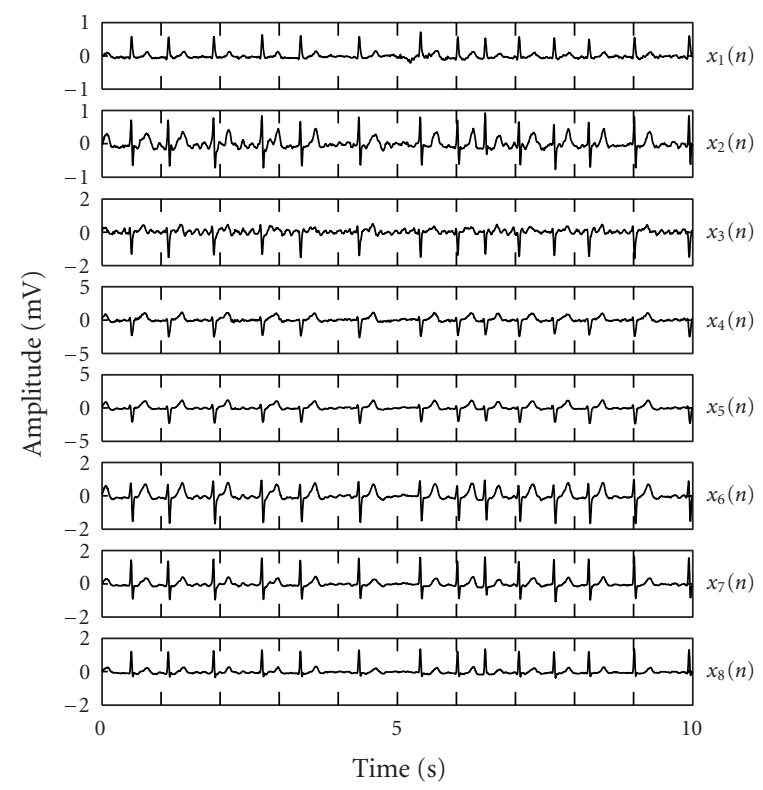

(a)

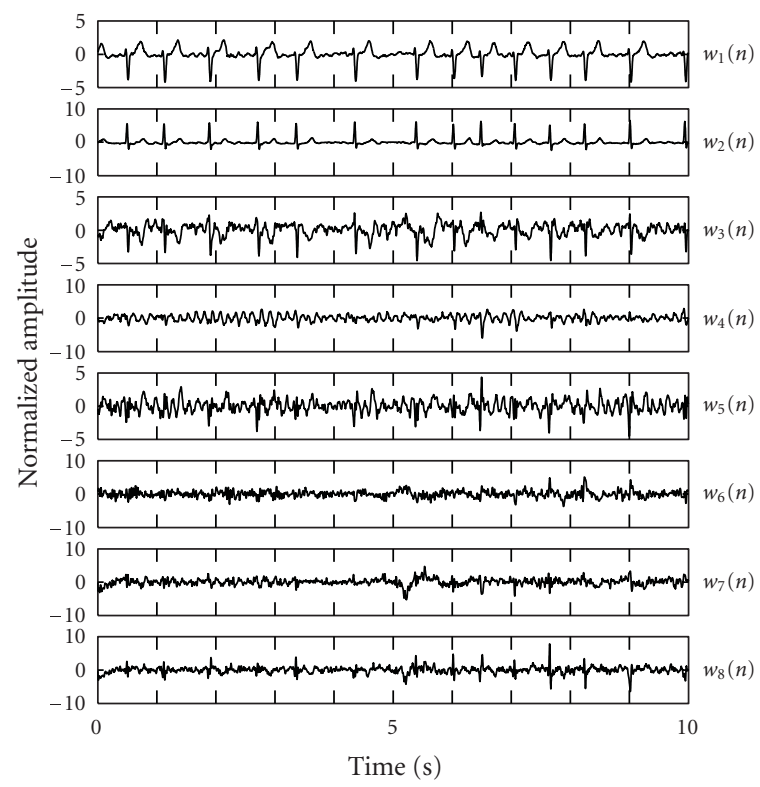

(b)

FIgURE 12: Example of atrial signal extraction during AF using a multilead PCA approach. (a) The original 8 ECG leads, where $x_{1}$ and $x_{2}$ are I and II limb leads, and $x_{3}$ to $x_{8}$ are the precordial leads, and (b) the corresponding principal components.

the observation of mixtures. Indeed, a BSS-based solution that not only exploits second-order statistics but also higherorder statistics to estimate the fibrillatory wave has been proposed [67].

So far, PCA has been applied to extract atrial signals for monitoring the effects of (1) antiarrhythmic drugs [63] and (2) linear atrial ablation [68]. After extraction of fibrillatory waves, FFT has been applied to detect the main frequency, which was shown to decrease with the administration of either amiodarone (from $5.8 \mathrm{~Hz}$ to $4.9 \mathrm{~Hz}$ ), flecainide (from $5.3 \mathrm{~Hz}$ to $4.7 \mathrm{~Hz}$ ), or sotalol (from $5.9 \mathrm{~Hz}$ to $4.9 \mathrm{~Hz}$ ) [63]. Similarly, fibrillatory frequency changes in response to linear left atrial ablation have been monitored and the effect on fibrillatory frequency of roof and mitral isthmus lines have been quantified [68]. Fibrillatory frequency decreased from $5.66 \mathrm{~Hz}$ to $5.15 \mathrm{~Hz}$ with a greater decrease after left atrial roof ablation compared with mitral isthmus ablation $(0.31 \mathrm{~Hz}$ versus $0.10 \mathrm{~Hz})$. Even though there was a trend to lower baseline frequencies with successful ablation, this study was not powered to predict outcome, although an invasive study supports this conclusion [69].

\section{BODY SURFACE POTENTIAL MAPPING}

Body surface potential mapping (BSPM) refers to the recording and analysis of temporal and spatial distributions of ECG potentials acquired multiple sites on the torso. In contrast to the analysis of the 12-lead ECG, where wave amplitudes, intervals, and morphology are usually considered, BSPM is rather considered in terms such as the shape of the potential distribution and the number and location of extrema. Since the electrodes that define such a map are closely spaced on the body surface, therefore containing considerable redundancy, PCA-based methods have been employed for data compression. It has been shown that spatial redundancy can be substantially reduced using the definition in $(28)[70,71]$, thereby resulting in a subset of leads which contains much richer information than subsets of the original leads of the same size. From such a subset of leads, better separation can be made of different types of patients $[72,73]$.

The analysis of a body surface potential map is particularly attractive and challenging since the map contain most of the electrocardiographic information that can be retrieved noninvasively. There is evidence that subjects at risk for ventricular tachycardia (VT) have unique map characteristics [74], for example, the spatial distribution of QRST integral has been found useful to stratify patients at risk for VT [75]. Results similar to those based on the QRST integral can be obtained for principal components obtained from (3), using either lead piling or basis functions forced to have the separable structure in (30). In both cases, susceptibility to VT can be predicted by the principal components [76], yielding results in terms of sensitivity and specificity similar to those based on the QRST integral [75]. Figure 13 illustrates the use of lead piling combined with (3), and the far less computationally demanding approach with separable basis functions. Figure 14 displays the basis functions $\mathbf{B}_{n, l}$ of order 14 that result from lead piling, reflecting that there is no exact temporal behavior from lead to lead, although a certain consistency can be observed. Figure 15 displays the basis functions $\mathbf{B}_{n, l}$ when the separable structure is assumed, using the correlation matrix estimates in (35); note the identical temporal behavior from lead to lead. The small differences between these two approaches are also reflected by the small differences found in clinical classification [76]. 


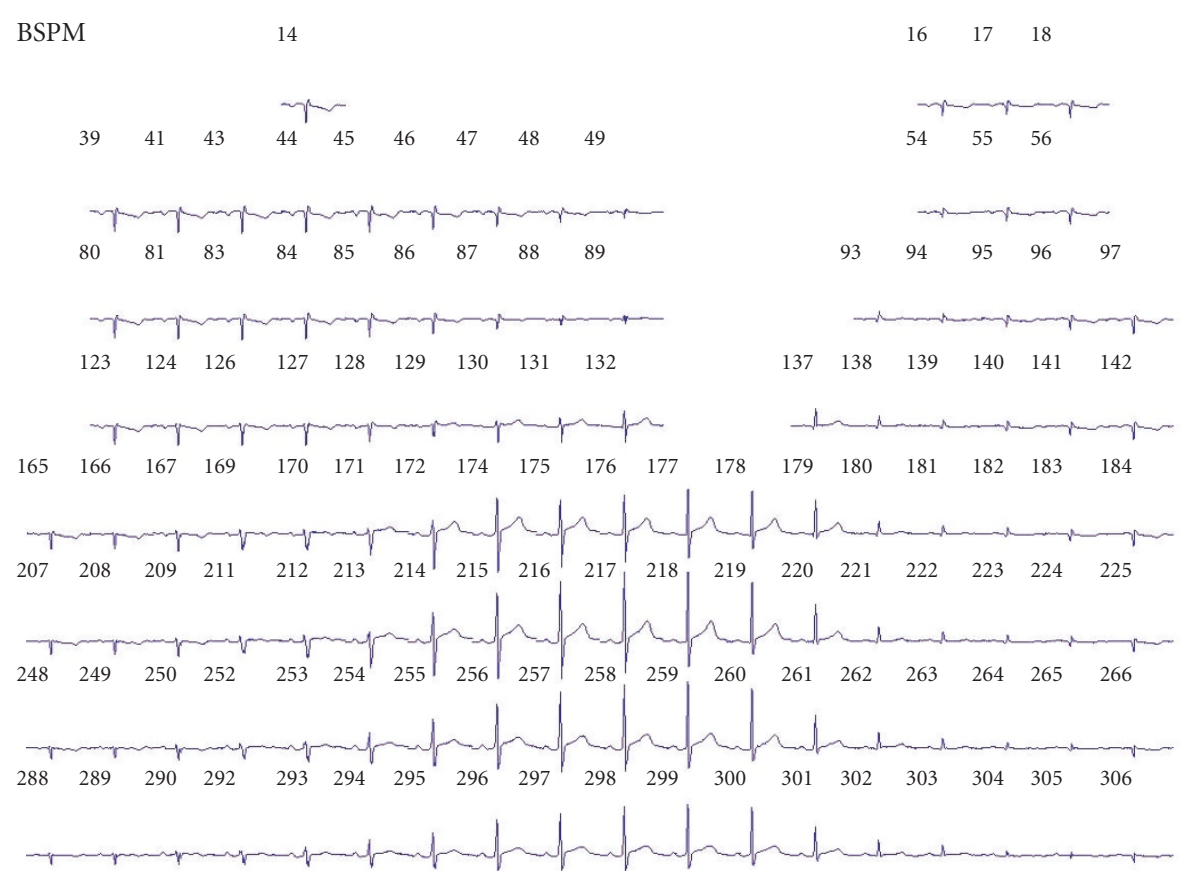

FIGURE 13: Data matrix $\mathbf{D}_{i}$ with one beat from a BSPM recording. The signal from lead $l$ is plotted around the torso location where the sensing electrode is located. The torso is displayed in an unfolded format, the right subplot corresponds to the back, the left one to the front, and the middle hole corresponds to the left axile.

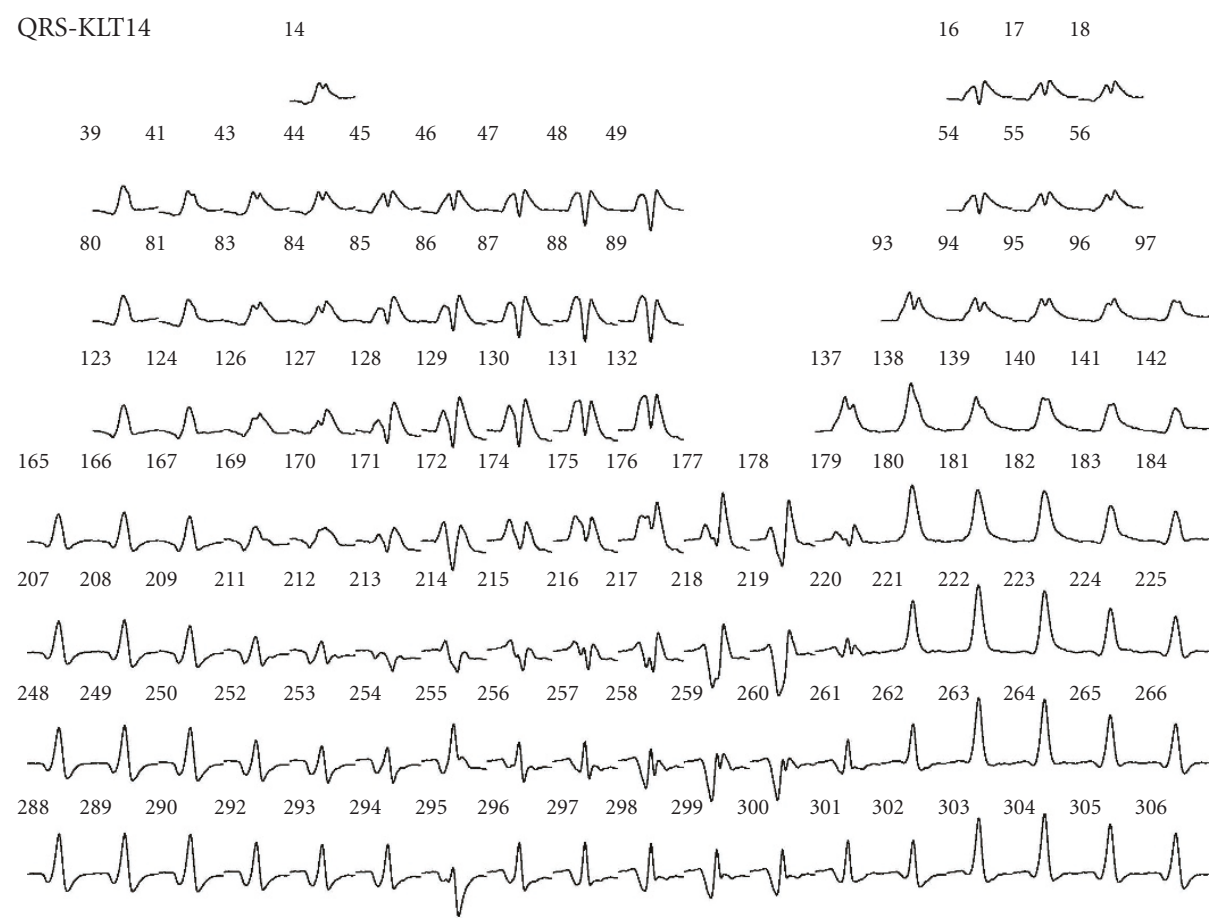

FIGURE 14: Basis function $\mathbf{B}_{n, l}$ derived from the BSPM data displayed in Figure 13, using lead piling ( $\mathbf{B}_{n, l}$ is obtained by depiling the eigenvector of order 14).

\section{CONCLUSIONS}

Several PCA-based strategies are available which exploit the fact that the ECG signal exhibits intrabeat, interbeat, and interlead redundancy. Although the underlying principle is the same in all ECG applications, the results are obtained and interpreted in quite diverse ways. In some applications, the goal is to find a more compact representation of the signal, 


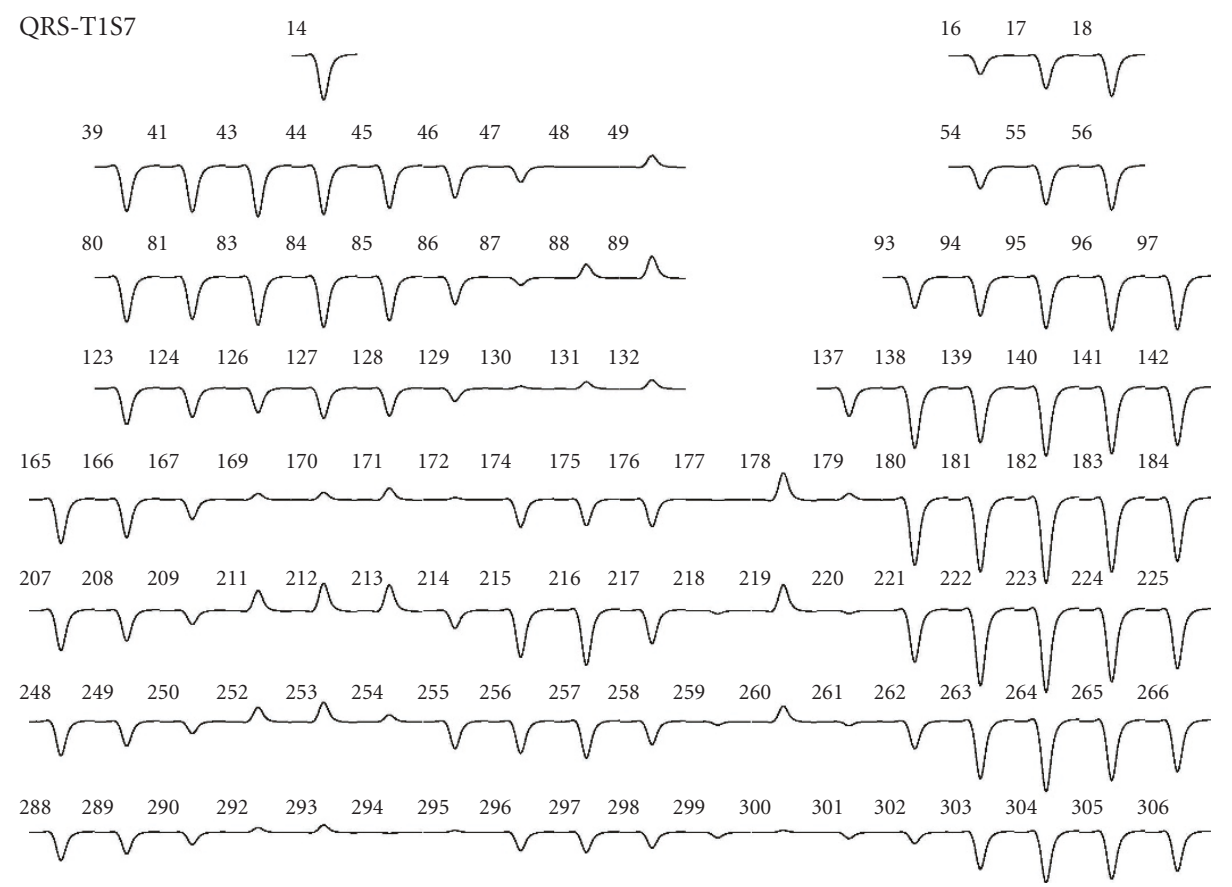

FIgURE 15: Basis function $\mathbf{B}_{n, l}=\mathbf{t}_{n} \mathbf{s}_{l}^{T}$ derived from the BSPM data displayed in Figure 13, assuming a separable structure of the time-lead correlation matrix $(n=1, l=7)$.

while in others it is to search for specific patterns or to extract a certain physiologic activity. In other applications, PCA may serve as a powerful, intermediate step when addressing problems related to noise reduction and beat classification. To date, PCA has been used to solve signal processing issues, most notably ECG data compression, as well as clinically oriented issues related to the characterization and diagnosis of myocardial ischemia, ventricular repolarization, and AF. In the future, PCA will continue to play an important role, for example, in electrical imaging of the heart-this application comprises large amounts of data which may call for methods that exploit all three types of signal redundancies.

\section{ACKNOWLEDGMENTS}

This project was supported by grants from the Swedish Science Foundation (2003-5176), TEC2004-05263-C02-02/ TCM from CICYT/FEDER Spain, TEC2005-08401-C02-01 from CICYT Spain, and CIBER CB06/01/0062. Travel grants were partially financed by the NordForsk Network "Electrocardiology in Atrial Fibrillation" and Universidad Politécnica de Valencia through its research incentive program.

\section{REFERENCES}

[1] I. T. Joliffe, Principal Component Analysis, Springer, New York, NY, USA, 2002.

[2] D. Callaerts, B. De Moore, J. Vandewalle, W. Sansen, G. Vantrappen, and J. Janssens, "Comparison of SVD methods to extract the foetal electrocardiogram from cutaneous electrode signals," Medical and Biological Engineering and Computing, vol. 28, no. 3, pp. 217-224, 1990.
[3] G. J. H. Uijen and A. van Oosterom, "The filtering of multilead ECGs by means of the singular value decomposition," in Proceedings of the 18th Annual Conference on Computers in Cardiology (CIC '91), pp. 137-140, Venice, Italy, September 1991.

[4] P. P. Kanjilal, S. Palit, and G. Saha, "Fetal ECG extraction from single-channel maternal ECG using singular value decomposition," IEEE Transactions on Biomedical Engineering, vol. 44, no. 1, pp. 51-59, 1997.

[5] B. Acar and H. Köymen, "SVD-based on-line exercise ECG signal orthogonalization," IEEE Transactions on Biomedical Engineering, vol. 46, no. 3, pp. 311-321, 1999.

[6] J. S. Paul, M. R. Reddy, and V. J. Kumar, "A transform domain SVD filter for suppression of muscle noise artefacts in exercise ECG's," IEEE Transactions on Biomedical Engineering, vol. 47, no. 5, pp. 654-663, 2000.

[7] L. De Lathauwer, B. De Moor, and J. Vandewalle, "SVD-based methodologies for fetal electrocardiogram extraction," in Proceedings of IEEE International Conference on Acoustics, Speech and Signal Processing (ICASSP '00), vol. 6, pp. 3771-3774, Istanbul, Turkey, June 2000.

[8] S. G. Priori, D. W. Mortara, C. Napolitano, et al., "Evaluation of the spatial aspects of T-wave complexity in the long-QT syndrome," Circulation, vol. 96, no. 9, pp. 3006-3012, 1997.

[9] P. M. Okin, R. B. Devereux, R. R. Fabsitz, E. T. Lee, J. M. Galloway, and B. V. Howard, "Principal component analysis of the $\mathrm{T}$ wave and prediction of cardiovascular mortality in American Indians: the strong heart study," Circulation, vol. 105, no. 6, pp. 714-719, 2002.

[10] L. Sörnmo and P. Laguna, Bioelectrical Signal Processing in Cardiac and Neurological Applications, Elsevier/Academic Press, Amsterdam, The Netherlands, 2005.

[11] N. Ahmed and K. R. Rao, Orthogonal Transforms for Digital Signal Processing, Springer, New York, NY, USA, 1975. 
[12] J. J. Gerbrands, "On the relationships between SVD, KLT and PCA," Pattern Recognition, vol. 14, no. 1-6, pp. 375-381, 1981.

[13] G. H. Golub and C. F. van Loan, Matrix Computations, The Johns Hopkins University Press, Baltimore, Md, USA, 2nd edition, 1989.

[14] S. Olmos, J. P. Martínez, and L. Sörnmo, "Spatio-temporal linear expansions for repolarization analysis," in Proceedings of Computers in Cardiology (CIC'02), vol. 29, pp. 689-692, Memphis, TN, USA, September 2002.

[15] T. Moon and W. Stirling, Mathematical Methods and Algorithms for Signal Processing, Prentice-Hall, Upper Saddle River, NJ, USA, 2000.

[16] P. Laguna, R. Jané, O. Meste, et al., "Adaptive filter for eventrelated bioelectric signals using an impulse correlated reference input: comparison with signal averaging techniques," IEEE Transactions on Biomedical Engineering, vol. 39, no. 10, pp. 1032-1044, 1992.

[17] P. Laguna, R. Jané, E. Masgrau, and P. Caminal, “The adaptive linear combiner with a periodic-impulse reference input as a linear comb filter," Signal Processing, vol. 48, no. 3, pp. 193203, 1996.

[18] F. H. Y. Chan, F. K. Lam, P. W. F. Poon, and W. Qiu, "Detection of brainstem auditory evoked potential by adaptive filtering," Medical and Biological Engineering and Computing, vol. 33, no. 1, pp. 69-75, 1995.

[19] V. Parsa and P. A. Parker, "Multireference adaptive noise cancellation applied to somatosensory evoked potentials," IEEE Transactions on Biomedical Engineering, vol. 41, no. 8, pp. 792800, 1994.

[20] C. A. Vaz and N. V. Thakor, "Adaptive Fourier estimation of time-varying evoked potentials," IEEE Transactions on Biomedical Engineering, vol. 36, no. 4, pp. 448-455, 1989.

[21] S. Olmos, L. Sörnmo, and P. Laguna, "Block adaptive filters with deterministic reference inputs for event-related signals: BLMS and BRLS," IEEE Transactions on Signal Processing, vol. 50, no. 5, pp. 1102-1112, 2002.

[22] D. J. Hamilton, W. A. Sandham, J. McQueen, and A. Blanco, "Electrocardiogram data compression by estimation of higher-index principal components," EURASIP Journal on Applied Signal Processing, vol. 5, no. 1, pp. 49-58, 1998.

[23] J. García, S. Olmos, G. B. Moody, R. G. Mark, and P. Laguna, "Adaptive estimation of Karhunen-Loève series applied to the study of ischemic ECG records," in Proceedings of Computers in Cardiology (CIC '96), pp. 249-252, Indianapolis, Ind, USA, September 1996.

[24] K. I. Diamantaras and S. Y. Kung, Principal Component Neural Networks: Theory and Applications, John Wiley \& Sons, New York, NY, USA, 1996.

[25] T. Stamkopoulos, K. Diamantaras, N. Maglaveras, and M. Strintzis, "ECG analysis using nonlinear PCA neural networks for ischemia detection," IEEE Transactions on Signal Processing, vol. 46, no. 11, pp. 3058-3067, 1998.

[26] S. Karlsson, "Representation of ECG records by KarhunenLoève expansions," in Proceedings of the 7th International Conference on Medical \& Biological Engineering, p. 105, Stockholm, Sweden, August 1967.

[27] N. Ahmed, P. J. Milne, and S. G. Harris, "Electrocardiographic data compression via orthogonal transforms," IEEE Transactions on Biomedical Engineering, vol. 22, no. 6, pp. 484-487, 1975.

[28] A. K. Evans, R. L. Lux, M. J. Burgess, R. F. Wyatt, and J. A. Abildskov, "Redundancy reduction for improved display and analysis of body surface potential maps. II. Temporal compression," Circulation Research, vol. 49, no. 1, pp. 197-203, 1981.
[29] M. E. Womble, J. S. Halliday, S. K. Mitter, M. C. Lancaster, and J. H. Triebwasser, "Data compression for storing and transmitting ECG's/VCG's," Proceedings of the IEEE, vol. 65, no. 5, pp. 702-706, 1977.

[30] R. Degani, G. Bortolan, and R. Murolo, "Karhunen-Loève coding of ECG signals," in Proceedings of Computers in Cardiology (CIC '90), pp. 395-398, Chicago, Ill, USA, September 1990.

[31] S. Olmos, M. Millán, J. García, and P. Laguna, "ECG data compression with the Karhunen-Loève transform," in Proceedings of Computers in Cardiology (CIC'96), pp. 253-256, Indianapolis, Ind, USA, September 1996.

[32] T. Blanchett, G. C. Kember, and G. A. Fenton, "KLT-based quality controlled compression of single-lead ECG," IEEE Transactions on Biomedical Engineering, vol. 45, no. 7, pp. 942945, 1998.

[33] D. J. Hamilton, J. McQueen, and W. A. Sandham, "Improved PCA-based electrocardiogram data compression using variable-length asymmetric beat vectors," EURASIP Journal on Applied Signal Processing, vol. 6, no. 4, pp. 194-202, 1999.

[34] P. Laguna, G. B. Moody, J. García, A. L. Goldberger, and R. G. Mark, "Analysis of the ST-T complex of the electrocardiogram using the Karhunen-Loève transform: adaptive monitoring and alternans detection," Medical and Biological Engineering and Computing, vol. 37, no. 2, pp. 175-189, 1999.

[35] Y. Zigel, A. Cohen, and A. Katz, "ECG signal compression using analysis by synthesis coding," IEEE Transactions on Biomedical Engineering, vol. 47, no. 10, pp. 1308-1316, 2000.

[36] Y. Zigel, A. Cohen, and A. Katz, "The weighted diagnostic distortion (WDD) measure for ECG signal compression," IEEE Transactions on Biomedical Engineering, vol. 47, no. 11, pp. 1422-1430, 2000.

[37] B. Bradie, "Wavelet packet-based compression of single lead ECG," IEEE Transactions on Biomedical Engineering, vol. 43, no. 5, pp. 493-501, 1996.

[38] A. E. Çetin, H. Köymen, and M. C. Aydin, "Multichannel ECG data compression by multirate signal processing and transform domain coding techniques," IEEE Transactions on Biomedical Engineering, vol. 40, no. 5, pp. 495-499, 1993.

[39] S. Olmos and P. Laguna, "Multi-lead ECG data compression with orthogonal expansions: KLT and wavelet packets," in Proceedings of Computers in Cardiology (CIC '99), pp. 539-542, Hannover, Germany, September 1999.

[40] F. J. Jager, R. G. Mark, G. Moody, and S. Divjak, "Analysis of transient ST segment changes during ambulatory monitoring using the Karhunen-Loève transform," in Proceedings of Computers in Cardiology (CIC '92), pp. 691-694, Durham, NC, USA, October 1992.

[41] J. García, P. Lander, L. Sörnmo, S. Olmos, G. Wagner, and P. Laguna, "Comparative study of local and Karhunen-Loèvebased ST-T indexes in recordings from human subjects with induced myocardial ischemia," Computers and Biomedical Research, vol. 31, no. 4, pp. 271-292, 1998.

[42] J. García, G. Wagner, L. Sörnmo, S. Olmos, P. Lander, and P. Laguna, "Temporal evolution of traditional versus transformed ECG-based indexes in patients with induced myocardial ischemia," Journal of Electrocardiology, vol. 33, no. 1, pp. 37-47, 2000.

[43] J. García, L. Sörnmo, S. Olmos, and P. Laguna, "Automatic detection of ST-T complex changes on the ECG using filtered RMS difference series: application to ambulatory ischemia monitoring," IEEE Transactions on Biomedical Engineering, vol. 47, no. 9, pp. 1195-1201, 2000. 
[44] J. García, G. Wagner, L. Sörnmo, P. Lander, and P. Laguna, "Identification of the occluded artery in patients with myocardial ischemia induced by prolonged percutaneous transluminal coronary angioplasty using traditional vs transformed ECG-based indexes," Computers and Biomedical Research, vol. 32, no. 5, pp. 470-482, 1999.

[45] J. García, M. Åström, J. Mendive, P. Laguna, and L. Sörnmo, "ECG-based detection of body position changes in ischemia monitoring," IEEE Transactions on Biomedical Engineering, vol. 50, no. 6, pp. 677-685, 2003.

[46] M. Zabel, M. Malik, K. Hnatkova, et al., "Analysis of T-wave morphology from the 12-lead electrocardiogram for prediction of long-term prognosis in male US veterans," Circulation, vol. 105, no. 9, pp. 1066-1070, 2002.

[47] H. C. Bazett, "An analysis of the time relations of electrocardiograms," Heart, vol. 7, pp. 353-370, 1920.

[48] E. Pueyo, P. Smetana, P. Caminal, A. Bayes de Luna, M. Malik, and P. Laguna, "Characterization of QT interval adaptation to RR interval changes and its use as a risk-stratifier of arrhythmic mortality in amiodarone-treated survivors of acute myocardial infarction," IEEE Transactions on Biomedical Engineering, vol. 51, no. 9, pp. 1511-1520, 2004.

[49] C. P. Day, J. M. McComb, and R. W. F. Campbell, "QT dispersion: an indication of arrhythmia risk in patients with long QT intervals," British Heart Journal, vol. 63, no. 6, pp. 342-344, 1990.

[50] M. Malik, B. Acar, Y. Gang, Y. G. Yap, K. Hnatkova, and A. J. Camm, "QT dispersion does not represent electrocardiographic interlead heterogeneity of ventricular repolarization," Journal of Cardiovascular Electrophysiology, vol. 11, no. 8, pp. 835-843, 2000.

[51] P. Smetana, V. N. Batchvarov, K. Hnatkova, A. J. Camm, and M. Malik, "Ventricular gradient and nondipolar repolarization components increase at higher heart rate," American Journal of Physiology: Heart and Circulatory Physiology, vol. 286, no. 1, pp. H131-H136, 2004.

[52] B. Acar, G. Yi, K. Hnatkova, and M. Malik, "Spatial, temporal and wavefront direction characteristics of 12-lead T-wave morphology," Medical and Biological Engineering and Computing, vol. 37, no. 5, pp. 574-584, 1999.

[53] P. D. Arini, E. R. Valverde, G. C. Bertran, and P. Laguna, "Geometrical and temporal ECG features for quantification of increased ventricular repolarization dispersion in an experimental heart rabbit model," in Proceedings of Computers in Cardiology (CIC '05), pp. 89-92, Lyon, France, September 2005.

[54] J. M. Smith, E. A. Clancy, C. R. Valeri, J. N. Ruskin, and R. J. Cohen, "Electrical alternans and cardiac electrical instability," Circulation, vol. 77, no. 1, pp. 110-121, 1988.

[55] J. P. Martínez and S. Olmos, "Methodological principles of T wave alternans analysis: a unified framework," IEEE Transactions on Biomedical Engineering, vol. 52, no. 4, pp. 599-613, 2005.

[56] P. Laguna, M. Ruiz, G. B. Moody, and R. G. Mark, "Repolarization alternans detection using the KL transform and the beatquency spectrum," in Proceedings of Computers in Cardiology (CIC '96), pp. 673-676, Indianapolis, Ind, USA, September 1996.

[57] V. Fuster, L. E. Rydén, R. W. Asinger, et al., "ACC/AHA/ESC guidelines for the management of patients with atrial fibrillation: executive summary a report of the American College of Cardiology/American Heart Association task force on practice guidelines and the European Society of Cardiology committee for practice guidelines and policy conferences (committee to develop guidelines for the management of patients with atrial fibrillation) developed in collaboration with the North American Society of pacing and electrophysiology," Circulation, vol. 104, no. 17, pp. 2118-2150, 2001.

[58] A. Bollmann, "First comes diagnosis then comes treatment: an underappreciated paradigm in atrial fibrillation management," European Heart Journal, vol. 26, no. 23, pp. 2487-2489, 2005.

[59] W. Einthoven, "Le télécardiogramme," Archives Internationales de Physiologie, vol. 4, pp. 132-164, 1906.

[60] A. Bollmann, N. K. Kanuru, K. K. McTeague, P. F. Walter, D. B. DeLurgio, and J. J. Langberg, "Frequency analysis of human atrial fibrillation using the surface electrocardiogram and its response to ibutilide," American Journal of Cardiology, vol. 81, no. 12, pp. 1439-1445, 1998.

[61] M. Holm, S. Pehrson, M. Ingemansson, et al., "Non-invasive assessment of the atrial cycle length during atrial fibrillation in man: introducing, validating and illustrating a new ECG method," Cardiovascular Research, vol. 38, no. 1, pp. 69-81, 1998.

[62] J. Slocum, A. Sahakian, and S. Swiryn, "Diagnosis of atrial fibrillation from surface electrocardiograms based on computerdetected atrial activity," Journal of Electrocardiology, vol. 25, no. 1, pp. 1-8, 1992.

[63] D. Raine, P. Langley, A. Murray, A. Dunuwille, and J. P. Bourke, "Surface atrial frequency analysis in patients with atrial fibrillation: a tool for evaluating the effects of intervention," Journal of Cardiovascular Electrophysiology, vol. 15, no. 9, pp. 10211026, 2004.

[64] F. Castells, C. Mora, J. J. Rieta, D. Moratal-Pérez, and J. Millet, "Estimation of atrial fibrillatory wave from single-lead atrial fibrillation electrocardiograms using principal component analysis concepts," Medical and Biological Engineering and Computing, vol. 43, no. 5, pp. 557-560, 2005.

[65] C. Mora, J. Castells, R. Ruiz, et al., "Prediction of spontaneous termination of atrial fibrillation using time frequency analysis of the atrial fibrillatory wave," in Proceedings of Computers in Cardiology (CIC '04), vol. 31, pp. 109-112, Chicago, Ill, USA, September 2004.

[66] A. Hyvärinen, J. Karhunen, and E. Oja, Independent Component Analysis, John Wiley \& Sons, New York, NY, USA, 2001.

[67] F. Castells, J. J. Rieta, J. Millet, and V. Zarzoso, "Spatiotemporal blind source separation approach to atrial activity estimation in atrial tachyarrhythmias," IEEE Transactions on Biomedical Engineering, vol. 52, no. 2, pp. 258-267, 2005.

[68] D. Raine, P. Langley, A. Murray, S. S. Furniss, and J. P. Bourke, "Surface atrial frequency analysis in patients with atrial fibrillation: assessing the effects of linear left atrial ablation," Journal of Cardiovascular Electrophysiology, vol. 16, no. 8, pp. 838-844, 2005.

[69] M. Haissaguerre, P. Sanders, M. Hocini, et al., "Changes in atrial fibrillation cycle length and inducibility during catheter ablation and their relation to outcome," Circulation, vol. 109, no. 24, pp. 3007-3013, 2004.

[70] R. L. Lux, A. K. Evans, M. J. Burgess, R. F. Wyatt, and J. A. Abildskov, "Redundancy reduction for improved display and analysis of body surface potential maps. I. Spatial compression," Circulation Research, vol. 49, no. 1, pp. 186-196, 1981.

[71] G. J. H. Uijen, A. Heringa, and A. van Oosterom, "Data reduction of body surface potential maps by means of orthogonal 
expansions," IEEE Transactions on Biomedical Engineering, vol. 31, no. 11, pp. 706-714, 1984.

[72] G. Kozmann, L. S. Green, and R. L. Lux, "Nonparametric identification of discriminative information in body surface maps," IEEE Transactions on Biomedical Engineering, vol. 38, no. 11, pp. 1061-1068, 1991.

[73] F. Kornreich, P. M. Rautaharju, J. W. Warren, B. M. Horacek, and M. Dramaix, "Effective extraction of diagnostic ECG waveform information using orthonormal basis functions derived from body surface potential maps," Journal of Electrocardiology, vol. 18, no. 4, pp. 341-350, 1985.

[74] M. J. Gardner, T. J. Montague, S. Armstrong, B. M. Horacek, and E. Smith, "Vulnerability to ventricular arrhythmia: assessment by mapping of body surface potential," Circulation, vol. 73, no. 4, pp. 684-692, 1986.

[75] C. L. Hubley-Kozey, L. B. Mitchell, M. J. Gardner, et al., "Spatial features in body-surface potential maps can identify patients with a history of sustained ventricular tachycardia," Circulation, vol. 92, no. 7, pp. 1825-1838, 1995.

[76] R. Bailón, S. Olmos, B. M. Horacek, and P. Laguna, "Identification of patients at risk for ventricular tachycardia by means of body surface potential maps," in Proceedings of the 30th Annual Computers in Cardiology (CIC '03), vol. 30, pp. 217-220, Thessaloniki, Greece, September 2003.

Francisco Castells was born in Valencia, Spain, in 1976. He received the M.Eng. degree in telecommunications engineering from the Universidad Politécnica de Valencia (UPV) in 2000. After working in the telecommunications manufacturing industry for Alcatel SEL AG in Germany (20002001), he received the Ph.D. degree in Electronics Engineering from the UPV in 2006. $\mathrm{He}$ is currently an Associate Lecturer at the

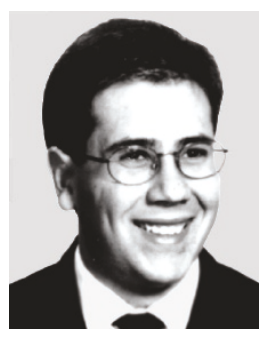
Department of Electronic Engineering, UPV, where he is also a member of the Bioengineering, Electronic and Telemedicine (BeT) Research Group. His research interests involve statistical signal processing of biomedical signals, with special emphasis on the characterization and analysis of atrial fibrillation.

Pablo Laguna was born in Jaca (Huesca), Spain in 1962. He received the Physics degree (M.S.) and the Doctor in Physic Science degree (Ph.D.) from the Science Faculty at the University of Zaragoza, Spain, in 1985 and 1990, respectively. The Ph.D. thesis was developed at the Biomedical Engineering Division of the Institute of Cybernetics (UPC-CSIC) under the direction of Pere Caminal. He is Full Professor of sig-

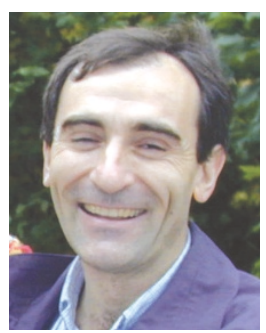
nal processing and communications in the Department of Electrical Engineering at the Engineering School, and a Researcher at the Aragón Institute for Engineering Research (I3A), both at University of Zaragoza, Spain. From 1992 to 2005, he was an Associate Professor at the same University and from 1987 to 1992, he worked as Assistant Professor of automatic control in the Department of Control Engineering at the Politecnic University of Catalonia (UPC), Spain, and as a Researcher at the Biomedical Engineering Division of the Institute of Cybernetics (UPC-CSIC). His professional research interests are in signal processing, in particular applied to biomedical applications.
He is, together with L. Sörnmo, the author of Bioelectrical Signal Processing in Cardiac and Neurological Applications (Elsevir, 2005)

Leif Sörnmo received the M.S. and Ph.D. degrees in electrical engineering from Lund University, Lund, Sweden, in 1978 and 1984, respectively. He held a research position at the Department of Clinical Physiology, Lund University, from 1983 to 1995, where he worked on computer-based ECG analysis. Since 1990, he has been with the Signal Processing Group, Department of Electroscience, Lund University, where he now

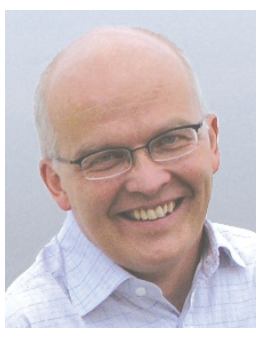
holds a position as Professor in biomedical signal processing. His main research interests include statistical signal processing and modeling of biomedical signals. Current research projects include methods for atrial fibrillation analysis, multimodal signal processing in hemodialysis, detection of otoacoustic emissions, and power efficient signal processing in implantable devices. He is, together with Pablo Laguna, the author of Bioelectrical Signal Processing in Cardiac and Neurological Applications (Elsevier, 2005). He has been Associate Editor of Computers in Biomedical Research (19972000). He is currently Associate Editor of the IEEE Transactions on Biomedical Engineering, and on the editorial boards of Journal of Electrocardiology, and Medical and Biological Engineering \& Computing.

Andreas Bollmann is Assistant Professor of medicine at Otto-von-Guericke-University Magdeburg, Germany. Since 1995, he has been with the Department of Cardiology at the University Hospital in Magdeburg, where he heads the noninvasive Electrocardiology Laboratories and the General Cardiology Outpatient Clinic. He has been a Research Scientist at Emory University, Atlanta, USA (1996-1997), Lund University,

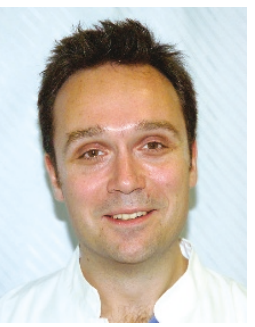
Sweden (from 2002 till now), Good Samaritan Hospital and Harbor-UCLA Medical Center, Los Angeles, USA (2003-2004, 2006). His research interests include atrial fibrillation, antiarrhythmic drugs and electrocardiology. Currently, he cochairs the NordForsk network "Electrocardiology in Atrial Fibrillation."

José Millet Roig was born in Valencia, Spain, in 1968. He received the M.S. degree in physics from the Universidad de Valencia (UV), and the Ph.D. degree in electrical and electronics engineering from the Universidad Politécnica de Valencia (UPV), in 1991 and 1997, respectively. He is currently an Associate Professor of electronics and biomedical signal processing at the Electronics Engineering Department, UPV.

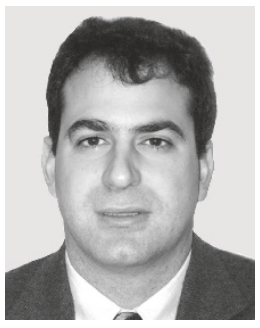
From 1991 to 1999, he worked as Assistant Professor in the same department. Since 1997, he is the coordinator of the Biomedical Engineering branch within the BET (Biomedical Engineering, Electronics and Telemedicine) Research Group of UPV. His professional research interests involve biomedical signal processing, biomedical signal acquisition and instrumentation, implantable devices for treatment of cardiac arrhythmias and Cardiac MRI. 